\title{
Groundwater Flow Processes and Human Impact along the Arid US-Mexican Border, Evidenced by Environmental Tracers: The Case of Tecate, Baja California
}

\author{
Jürgen Mahlknecht ${ }^{1, *(\mathbb{D})}$, Luis Walter Daessle ${ }^{2}$, Maria Vicenta Esteller ${ }^{3}$, \\ Juan Antonio Torres-Martinez ${ }^{1}$ and Abrahan Mora ${ }^{1}$ \\ 1 Escuela de Ingeniería y Ciencias, Tecnológico de Monterrey, Av. Eugenio Garza Sada Sur No. 2501, \\ Monterrey CP 64849, Nuevo León, Mexico; ja.torres@itesm.mx (J.A.T.-M.); abrahanmora@itesm.mx (A.M.) \\ 2 Instituto de Investigaciones Oceanológicas, Universidad Autónoma de Baja California, Carretera \\ Transpeninsular Tijuana-Ensenada No. 3917, Fraccionamiento Playitas, Ensenada CP 22860, Baja California, \\ Mexico; walter@uabc.edu.mx \\ 3 Centro Interamericano de Recursos del Agua (CIRA), Facultad de Ingeniería, \\ Universidad Autónoma del Estado de México, Cerro de Coatepec, Ciudad Universitaria, Toluca CP 50110, \\ Estado de México, Mexico; mvestellera@uaemex.mx \\ * Correspondence: jurgen@itesm.mx; Tel.: +52-81-8155-2573
}

Received: 19 March 2018; Accepted: 27 April 2018; Published: 30 April 2018

\begin{abstract}
With the increasing population, urbanization and industry in the arid area of Tecate, there is a concomitant increase in contaminants being introduced into the Tecate River and its aquifer. This contamination is damaging the usable groundwater supply and making local residents and commercial enterprises increasingly dependent on imported water from the Colorado River basin. In this study we apply a suite of chemical and isotopic tracers in order to evaluate groundwater flow and assess contamination trends. Groundwater recharge occurs through mountain-block and mountain-front recharge at higher elevations of the ranges. Groundwater from the unconfined, alluvial aquifer indicates recent recharge and little evolution. The increase in salinity along the flow path is due to interaction with weathering rock-forming silicate minerals and anthropogenic sources such as urban wastewater, residual solids and agricultural runoff from fertilizers, livestock manure and/or septic tanks and latrines. A spatial analysis shows local differences and the impact of the infiltration of imported waters from the Colorado River basin. The general trend of impaired water quality has scarcely been documented in the last decades, but it is expected to continue. Since the groundwater system is highly vulnerable, it is necessary to protect groundwater sources.
\end{abstract}

Keywords: groundwater geochemistry; environmental isotopes; groundwater contamination; arid climate; Tecate; Baja California; Mexico

\section{Introduction}

The peninsula of Baja California in northwestern Mexico has few available water resources and is one of the most arid areas in Mexico. The Tecate River is a sub-basin of the Tijuana River basin, a coastal watershed in Baja California adjacent to the USA-Mexico international border that discharges into the Pacific Ocean (Figure 1). In 2000, the groundwater resources provided about thirty percent of Tecate's potable water supply [1], decreasing to only twenty percent in 2015 [2]. In addition, with the increase in population, urbanization, and industry in Tecate, there is a concomitant increase in contaminants being introduced into the Tecate River and groundwater resources. The Tecate River, 
which under natural conditions is an ephemeral stream, has become a perennial one with poor water quality downstream of the discharges. Major point sources of pollution to the river include the discharge of poorly treated sewage from the Tecate municipal plant, the discharge of waters containing high organic matter from a brewery, and effluent discharge from a manufacturing plant complex which includes a large metal-working industry and the effluent from a slaughterhouse [3,4]. Further, it is thought that the aquifer is being degraded by leaking septic tanks, underground storage containers holding fuel products and other chemicals, and agricultural run-off [5].

This contamination is reducing the usable groundwater supply, making local residents and commercial enterprises more dependent on imported water from the Colorado River basin through the 130-km Rio Colorado-Tijuana Aqueduct [2,6]. The volume of water imported from the Colorado basin is susceptible to international agreements, depending on water availability in the USA, and also to climate change factors [7]. The annual extraction of groundwater amounts to ca. 12 million $\mathrm{m}^{3}$, of which $35.7 \%$ is utilized for public-urban use, $23.5 \%$ for agricultural use, $4.3 \%$ for livestock use, $16.9 \%$ for industrial use, $0.9 \%$ for services, and $18.7 \%$ for multiple uses [2].

Previous studies in the Tecate aquifer agree that, in general, groundwater was of good quality in terms of salinity (TDS $=300-1200 \mathrm{mg} \mathrm{L}^{-1}$ ). The water type has been described as mixed with a tendency towards bicarbonates [8]. Available information on groundwater in two wells ca. $50 \mathrm{~km}$ East from Tecate indicates that TDS and sulfate concentrations were around $1200 \mathrm{mg} \mathrm{L}^{-1}$ and $450 \mathrm{mg} \mathrm{L}^{-1}$, respectively, with a total hardness of up to $716 \mathrm{mg} \mathrm{L}^{-1}$, which could be associated with the local presence of limestone deposits and a limestone quarry [9].

The Tecate area has a rugged landscape with small intermountain valleys where groundwater storage is small in size [10]. Relatively little information is available about its hydrogeological settings, well construction, and water table configuration. Thus, in the present paper we develop a cost-effective method for evaluating groundwater quality and dynamics, which may help water managers in decision making and create higher awareness among local stakeholders about local water resources and their management.

Mathematical tools based on hydraulic data are an excellent way to understand groundwater dynamics and chemical transport. They are however normally time-consuming and expensive. Thus, we propose an alternative using environmental tracers. Chemical constituents in groundwater are not only useful to characterize the chemistry and to evaluate the water quality, but also to understand local trends, to identify hydrochemical processes and to predict the evolution of groundwater chemistry. Isotope ratios help to study the origin and transformation processes in groundwater. Stable isotopes of the water molecule, deuterium $\left({ }^{2} \mathrm{H}\right)$ and oxigen-18 $\left({ }^{18} \mathrm{O}\right)$, serve for hydrological fingerprinting.

Radioactive isotopes are useful to evaluate groundwater residence times and eventually to find the relation between these and groundwater vulnerability. Tritium $\left({ }^{3} \mathrm{H}\right)$ is an environmental pulse tracer derived from the atmosphere that is used as a dating tool for young water, i.e., for timescales of 50 years or less [11,12]. Dating with ${ }^{3} \mathrm{H}$ is based on knowledge of the local input function and the measured concentration of ${ }^{3} \mathrm{H}$ in the groundwater. The ${ }^{3} \mathrm{H}$ concentration in the atmosphere-and thus in young groundwater-recently decreased in North America from its maximum value at the bomb-tritium peak of the 1960s, ultimately reaching pre-bomb values around 1992 [13].

Radiocarbon $\left({ }^{14} \mathrm{C}\right)$ is the leading tool for estimating the age of paleo groundwater and fossil groundwater, which can be used to date samples that are hundreds of years to approximately 35,000 years old $[14,15]$. Radiocarbon dating is usually performed by measuring the loss of the parent radionuclide in the dissolved inorganic carbon of a given groundwater sample. Because chemical reactions and the evolution of carbonate systems may dilute the initial ${ }^{14} \mathrm{C}$ activity in the dissolved inorganic carbon (DIC), the age must be adjusted geochemically $[14,16,17]$. Carbon-13-mixing models are very useful in correcting ${ }^{14} \mathrm{C}$ ages. These adjustment models use measured carbon- $13\left({ }^{13} \mathrm{C}\right)$, combined with ${ }^{14} \mathrm{C}$ activity and a series of other parameters (e.g., alkalinity, $\mathrm{pH}$, and temperature) $[18,19]$. 
In the present study these tools are applied all together as a suite $[20,21]$, in order to evaluate groundwater flow patterns, groundwater residence times and to assess contamination trends and aquifer vulnerability to surface sources.

\section{Study Area}

Overall, rainfall in Tecate is scarce and cyclonic, occurring during the winter season from December to March, while the dry season is from May to September (a cold semi-arid climate, according to Köppen). The average yearly temperature for the Tecate catchment is $16.5^{\circ} \mathrm{C}$, with a gradient from 15.0 (east) to $18.0^{\circ} \mathrm{C}$ (west). The average annual precipitation and evapotranspiration (Turc formula) are $321 \mathrm{~mm}$ and $317 \mathrm{~mm}$, respectively [8,22].

Groundwater recharge to the Tecate aquifer is mainly driven by local rainfall and runoff from the Laguna Mountains (USA) and the Sierra Juarez (Mexico) which form part of the Peninsular Range. An estimate of the annual water balance for this aquifer by [8] suggests $10.1 \mathrm{Mm}^{3}$ natural plus induced recharge and $11.8 \mathrm{Mm}^{3}$ discharge, resulting in a water deficit of $-1.7 \mathrm{Mm}^{3}$. The mountain-front type of groundwater recharge is typical for valley aquifers in mountainous watersheds in arid and semi-arid regions $[23,24]$.

The rocks of the area are grouped into two hydrogeological units, constituting of a permeable and an impervious unit [8] (Figure 1). The first one consists of granular fragments of fluvial and alluvial origin. The fluvial deposits consist of gravels, sands and clays in smaller quantities, located mainly along the streambeds. The lower, impermeable unit is composed of metamorphic and intrusive igneous rocks—such as granite and diorite- that cover most of the area [8]. These igneous rocks are altered and fractured by weathering processes, representing the development of a secondary permeability. This unit serves as a lateral boundary for the aquifer and transmits the precipitation to the permeable unit, but its water storage is insignificant.
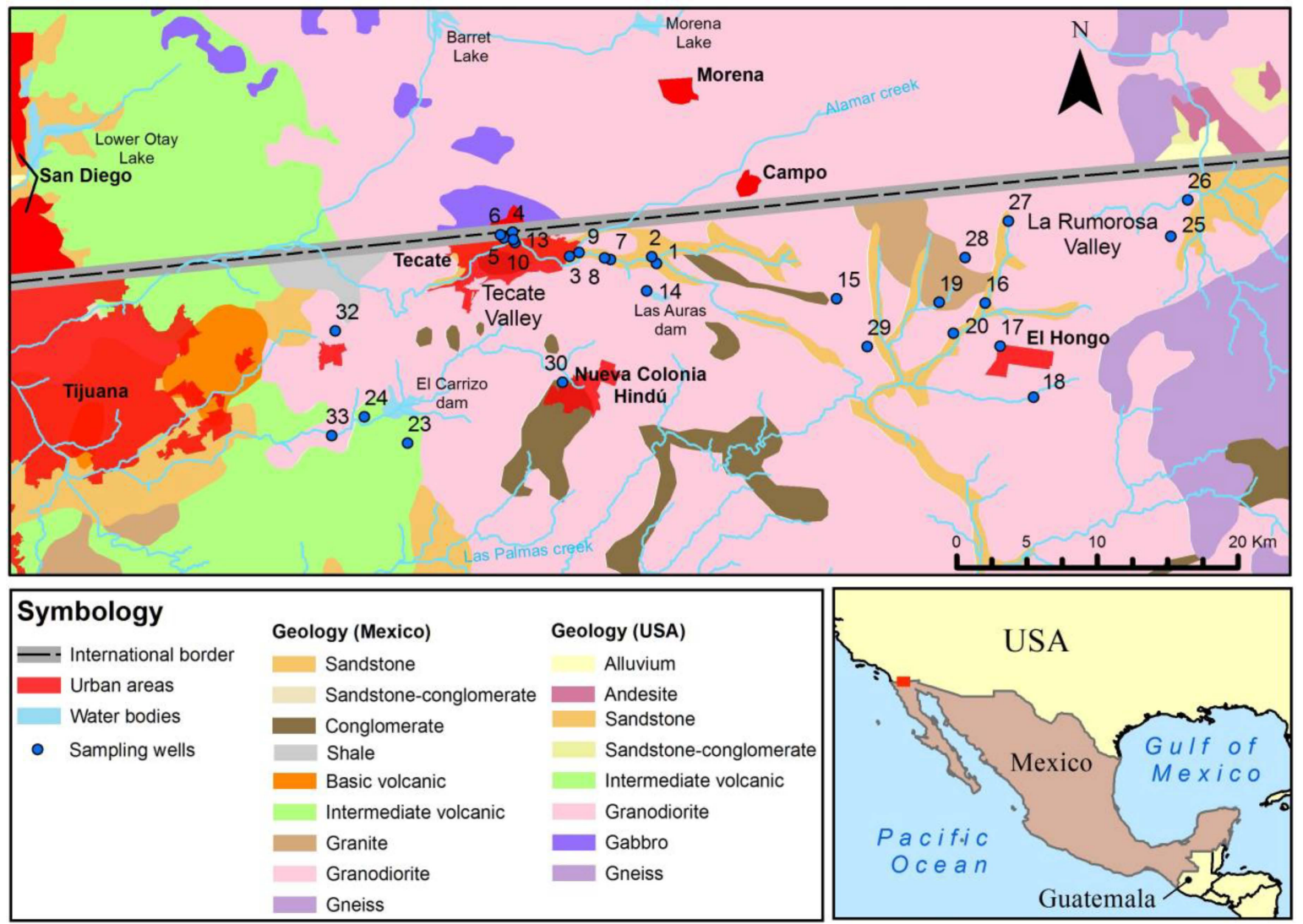

Figure 1. Map of the study area showing the location of the sampled wells. 
The aquifer system comprises metamorphic and igneous intrusive rocks (granodiorite, tonalite and diorite), overlain by discordant outcrops of continental clastic rocks (alluvial sediments) $[9,25,26]$. The aquifer is unconfined and has an approximate thickness of 12-18 $\mathrm{m}$ [10], with a moderate to high permeability.

Granodiorite contains principally quartz, plagioclase, and biotite. Tonalite contains quartz $\mathrm{SiO}$, andesine $(\mathrm{Na}, \mathrm{Ca})(\mathrm{Si}, \mathrm{Al})_{4} \mathrm{O}_{8}$, and small amounts of orthoclase $\left(\mathrm{KAlSi}_{3} \mathrm{O}_{8}\right)$. Diorite contains $2 / 3$ plagioclase $(\mathrm{Na}, \mathrm{Ca})(\mathrm{Si}, \mathrm{Al})_{3} \mathrm{O}_{8}$ and $1 / 3$ mafic minerals (hornblende $\mathrm{Ca}_{2}(\mathrm{Mg}, \mathrm{Fe}, \mathrm{Al}) 5(\mathrm{Al}, \mathrm{Si})_{8} \mathrm{O}_{22}(\mathrm{OH})_{2}$, biotite $\mathrm{K}(\mathrm{Mg}, \mathrm{Fe})_{3} \mathrm{AlSi}_{3} \mathrm{O}_{10}(\mathrm{OH}, \mathrm{F})_{2}$, pyroxene $\left.(\mathrm{Ca}, \mathrm{Mg}, \mathrm{Fe}, \mathrm{Mn}, \mathrm{Na}, \mathrm{Li})(\mathrm{Al}, \mathrm{Mg}, \mathrm{Fe}, \mathrm{Mn}, \mathrm{Cr}, \mathrm{Sc}, \mathrm{Ti})(\mathrm{Si}, \mathrm{Al})_{2} \mathrm{O}_{6}\right)$. Chemically, this indicates a relatively homogenous aquifer system with predominantly silicate weathering [2].

\section{Materials and Methods}

\subsection{Field Work and Laboratory}

Twenty-six wells were sampled in December 2014 for major and minor chemical constituents $\left(\mathrm{Na}^{+}, \mathrm{K}^{+}, \mathrm{Ca}^{2+}, \mathrm{Mg}^{2+}, \mathrm{HCO}_{3}^{-}, \mathrm{Cl}^{-}, \mathrm{SO}_{4}^{2-}, \mathrm{NO}_{3}^{-}, \mathrm{F}^{-}, \mathrm{Sr}^{2+}, \mathrm{Si}, \mathrm{Fe}\right)$, stable isotope ratios $\left({ }^{2} \mathrm{H},{ }^{18} \mathrm{O},{ }^{13} \mathrm{C}\right.$ in DIC), as well as isotopic activity $\left({ }^{3} \mathrm{H},{ }^{14} \mathrm{C}\right.$ in DIC). Physicochemical parameters were measured in situ and recorded after the variables had stabilized. A pre-calibrated portable multi-meter (Orion Star A329, Thermo Fisher Scientific, Waltham, MA, USA) was used for electrical conductivity (EC), temperature (T), $\mathrm{pH}$ and dissolved oxygen (DO). Alkalinity was determined in the field by titration with $0.02 \mathrm{~N} \mathrm{H}_{2} \mathrm{SO}_{4}$. Water samples were collected and stored in HDPE bottles at $4{ }^{\circ} \mathrm{C}$ and then sent to the laboratory for analysis within a week. Aliquots for cations were filtered through $0.45 \mu \mathrm{m}$ cellulose acetate membrane and acidified through addition of high purity nitric acid $(\mathrm{pH} \leq 2)$. Dissolved cations and anions were measured using inductively coupled plasma mass spectrometry (ICP-MS) and ion chromatography (IC), respectively, following standard methods [27].

Samples analyzed for their $\delta^{18} \mathrm{O}$ and $\delta^{2} \mathrm{H}$ ratios were collected in $100 \mathrm{~mL}$ glass bottles and measured with an isotope ratio mass spectrometer (MM 903; VG Isogas Ltd., Middlewich, UK), using the $\mathrm{H}_{2} \mathrm{O}-\mathrm{H}_{2}$ equilibration method $\left({ }^{2} \mathrm{H}\right)$, which has an analytical precision of $\pm 1.0 \%$, and the $\mathrm{H}_{2} \mathrm{O}-\mathrm{CO}_{2}$ equilibration method $\left({ }^{18} \mathrm{O}\right)$, which has an analytical precision of $\pm 0.15 \%$. These measurements were performed at the Environmental Isotope Laboratory of the Department of Geosciences, University of Arizona. The results were reported as $\delta$-values with respect to Vienna Standard Mean Ocean Water (VSMOW).

Tritium, ${ }^{14} \mathrm{C}$-DIC and ${ }^{13} \mathrm{C}$-DIC were measured in 19 out of 26 samples. Tritium was analyzed at the Environmental Isotope Laboratory, University of Arizona (Tucson, AZ, USA). The electrolytical enrichment method used was similar to that of Östlund and Werner [28]. Counting was performed using a Quantulus 1220 liquid scintillation spectrophotometer (PerkinElmer, Waltham, MA, USA). Tritium concentrations are reported in tritium units (TU), in which $1 \mathrm{TU}$ is equal to one tritium atom in $10^{18}$ hydrogen atoms. The detection limit was $0.3 \mathrm{TU}$ and the analytical precision was typically better than $\pm 10 \%$. Radiocarbon activity was measured by accelerator mass spectrometry at the NSF-Arizona Accelerator Facility for Radioisotope Analysis (Tucson, AZ, USA) and reported as percent modern carbon (pMC). The analytical precision (1- $\sigma)$ ranged from $0.1 \mathrm{pMC}$ (for pMC values near zero) to 0.5 pMC (for pMC values near 100 pMC). Carbon-13 measurements were performed using a Finnigan Delta S spectrometer (Thermo Fisher Scientific, Waltham, MA, USA) at Environmental Isotope Laboratory, University of Arizona (Tucson, AZ, USA), on carbon dioxide previously released by acidifying sample water [29]. These results are reported in $\delta^{13} C_{\text {DIC }}$ with respect to the Vienna Pee Dee Belemnite (VPDB) standard and with an analytical precision of $0.15 \%(1-\sigma)$.

\subsection{Interpretation}

Samples with a chemical charge balance error $>10 \%$ were discarded for interpretation. The significant difference of mean values between this sampling campaign and a sampling campaign 
in 1981, was statistically evaluated by the $t$-test. The null hypothesis adopted in the $t$-test was that concentration levels of a particular solute were not significantly different between the years. This hypothesis was rejected when calculated $p$-values were less than 0.05 .

The water classification was done with a Hierarchical Cluster Analysis (HCA) scheme, considering 18 variables measured in all samples $\left(\mathrm{T}, \mathrm{pH}, \mathrm{EC}, \mathrm{OD}, \mathrm{Na}^{+}, \mathrm{K}^{+}, \mathrm{Ca}^{2+}, \mathrm{Mg}^{2+}, \mathrm{HCO}_{3}^{-}, \mathrm{Cl}^{-}, \mathrm{SO}_{4}^{2-}, \mathrm{NO}_{3}^{-}\right.$, $\mathrm{F}^{-}, \mathrm{Sr}^{2+}, \mathrm{Si}, \mathrm{Fe},{ }^{2} \mathrm{H},{ }^{18} \mathrm{O}$ ). Iteratively neighboring points (samples) using Ward's linkage rule were linked through a similarity matrix [30]. The squared Euclidian distance was selected as the similarity measurement. For the cluster analysis, log-normal distributed data were previously log-transformed, and all of the variables standardized ( $\mathrm{z}$ scores).

Saturation indexes were calculated in order to elucidate the equilibrium state of water with respect to minerals. The saturation index (SI) is defined as $\mathrm{SI}=\log (\mathrm{IAP} / \mathrm{K})$ where IAP is the ion activity product and $\mathrm{K}$ is the equilibrium constant. If $\mathrm{SI}=0$, the solution is in equilibrium with the mineral phase and no reaction is expected. If $\mathrm{SI}>0$, groundwater is oversaturated with respect to the particular mineral, which means that the mineral phase may precipitate to achieve equilibrium. Finally, if $\mathrm{SI}<0$, groundwater is undersaturated with the mineral phase, which means that dissolution is required to reach equilibrium. The PHREEQC program [31] was used for the calculation of saturation indexes.

Unadjusted ${ }^{14} \mathrm{C}$ ages were calculated from measured ${ }^{14} \mathrm{C}$ activities in DIC by using the Libby half-life and assuming initial ${ }^{14} \mathrm{C}$ activity of $100 \%$ modern carbon (pMC). Age adjustments were made using the Fontes \& Garnier and Pearson model $[18,32]$. This model considers a two-stage evolution of recharge waters, accounting for the dissolution and isotopic exchange of carbonate minerals with $\mathrm{CO}_{2}$ in the unsaturated zone, as well as isotopic exchange with carbonate rocks in the saturated zone $[14,21,33,34]$. The ${ }^{14} \mathrm{C}$ activity and $\delta^{13} \mathrm{C}$ value of dissolved carbonate minerals were set as $0 \mathrm{pMC}$ and $0 \%$ VPDB, respectively. The ${ }^{14} \mathrm{C}$ activity of soil $\mathrm{CO}_{2}$ in all samples is set to $102 \mathrm{pMC}$, as it appears to be the ${ }^{14} \mathrm{C}$ activity in samples from recharge areas [2]. The $\delta^{13} \mathrm{C}$ value of soil $\mathrm{CO}_{2}$ was set according to predominant natural vegetation in recharge areas. Tecate's vegetation includes coastal sage scrub, chaparral, pine forest, oak forest, and desert scrub. Also important are meadowlands, aquatic and riparian areas [35]. Thus, the $\delta^{13} \mathrm{C}$ value is set as $-24 \%$ VPDB to account for the mainly C3 plants in the recharge area. Isotopic fractionation factors were calculated using the methods of Vogel et al. [36]; Mook et al. [37]; and Deines et al. [38].

\section{Results}

\subsection{Chemical Characterization}

Table 1 presents the results for the physicochemical parameters, the chemical concentrations, stable isotope ratios and radioactive isotope activities of the groundwaters studied. The $\mathrm{pH}$ range is 7.0-8.6 (average $7.6 \pm 0.3$ ), the temperature is $13.6-21.8^{\circ} \mathrm{C}$ (average $18.4 \pm 2.1{ }^{\circ} \mathrm{C}$ ) and EC is $437-7480 \mu \mathrm{S} \mathrm{cm}^{-1}$ (average $1427 \pm 1326 \mu \mathrm{S}$ ). Dissolved oxygen varies between 2.8 and $13.1 \mathrm{mg} \mathrm{L}^{-1}$ (average $6.8 \mathrm{mg} \pm 2.5 \mathrm{mg} \mathrm{L}^{-1}$ ), indicating oxic conditions in groundwater. The dominance of cations is $\mathrm{Na}^{+}>\mathrm{Ca}^{2+}>\mathrm{Mg}^{2+}>>\mathrm{K}^{+}$and of anions is $\mathrm{HCO}_{3}^{-}>\mathrm{Cl}^{-}>\mathrm{SO}_{4}^{2-}>\mathrm{NO}_{3}^{-}>>\mathrm{F}^{-}$. The concentrations of $\mathrm{Cl}^{-}, \mathrm{NO}_{3}^{-}, \mathrm{SO}_{4}^{2-}$ and $\mathrm{F}^{-}$range from 34 to $1584 \mathrm{mg} \mathrm{L}^{-1}, 0.2$ to $35.5 \mathrm{mg} \mathrm{L}^{-1}, 12.6$ to $574.8 \mathrm{mg} \mathrm{L}^{-1}$ and 0.3 to $2.2 \mathrm{mg} \mathrm{L}^{-1}$, respectively. The higher values are found to the west (Tecate Valley) and to the extreme east (Rumorosa Valley) of the study area (Figure 1).

Seventy-seven percent of the samples have a TDS $>500 \mathrm{mg} \mathrm{L}^{-1}$, and $31 \%$ have TDS $>1000 \mathrm{mg} \mathrm{L}^{-1}$. This means that every third sample can be classified as slightly saline water. Gangster et al. reports an increasing deterioration in water quality starting in 1992 [35]. From previous studies for the study area, only one reports groundwater water chemical parameters (INEGI, 1982). This study considers a field sampling campaign in winter 1981, with 16 sites that match this study. Thus, it was possible to compare mean values between 1981 and 2014. 
Table 1. Physicochemical parameters, chemical concentrations, charge balance error, stable isotope ratios and isotopic activities.

\begin{tabular}{|c|c|c|c|c|c|c|c|c|c|c|c|c|c|c|c|c|c|c|c|c|c|c|}
\hline Well ID & Temp & $\mathrm{pH}$ & EC & OD & $\mathrm{Na}$ & $\mathrm{K}$ & $\mathrm{Ca}$ & $\mathrm{Mg}$ & $\mathrm{HCO}_{3}$ & $\mathrm{Cl}$ & $\mathrm{SO}_{4}$ & $\mathrm{NO}_{3}$ & $F$ & $\mathrm{Sr}$ & $\mathrm{Fe}$ & $\mathrm{Si}$ & Charge Balance Error & ${ }^{18} \mathrm{O}$ & ${ }^{2} \mathrm{H}$ & ${ }^{3} \mathrm{H}$ & ${ }^{13} \mathrm{C}$ & ${ }^{14} \mathrm{C}$ \\
\hline$(-)$ & $\left({ }^{\circ} \mathrm{C}\right)$ & (std. Unit) & $\left(\mu \mathrm{S} \mathrm{cm}^{-1}\right)$ & $\left(\mathrm{mg} \mathrm{L}^{-1}\right)$ & $\left(\mathrm{mg} \mathrm{L}^{-1}\right)$ & $\left(\mathrm{mg} \mathrm{L}^{-1}\right)$ & $\left(\mathrm{mg} \mathrm{L}^{-1}\right)$ & $\left(\mathrm{mg} \mathrm{L}^{-1}\right)$ & $\left(\mathrm{mg} \mathrm{L}^{-1}\right)$ & $\left(\mathrm{mg} \mathrm{L}^{-1}\right)$ & $\left(\mathrm{mg} \mathrm{L}^{-1}\right)$ & $\left(\mathrm{mg} \mathrm{L}^{-1}\right)$ & $\left(\mathrm{mg} \mathrm{L}^{-1}\right)$ & $\left(\mathrm{mg} \mathrm{L}^{-1}\right)$ & $\left(\mathrm{mg} \mathrm{L}^{-1}\right)$ & $\left(\mathrm{mg} \mathrm{L}^{-1}\right)$ & $(\%)$ & $(\%)$ & $(\%)$ & (TU) & $(\%)$ & (pMC) \\
\hline 1 & 19.9 & 7.7 & 706 & 5.0 & 76.1 & 2.5 & 56.1 & 23.8 & 414.3 & 65.1 & 31.3 & 1.0 & $<0.5$ & 0.264 & 0.010 & 9.2 & -6.8 & -6.84 & -46.47 & 72.2 & -13.0 & 107.2 \\
\hline 2 & 20.5 & 7.9 & 1111 & 7.2 & 149.0 & 6.0 & 61.6 & 37.4 & 604.8 & 123.5 & 56.2 & 6.6 & 0.7 & 0.422 & 0.010 & 10.2 & -6.8 & -6.40 & -43.75 & & - & - \\
\hline 3 & 18.7 & 7.8 & 1323 & 6.7 & 179.2 & 5.7 & 83.9 & 40.6 & 444.7 & 146.1 & 130.4 & 5.5 & 0.6 & 0.550 & 0.010 & 17.3 & 4.7 & -6.50 & -43.94 & & -13.4 & 104.0 \\
\hline 4 & 21.2 & 7.8 & 835 & 7.3 & 79.0 & 6.1 & 68.7 & 28.5 & 305.7 & 90.7 & 75.4 & 3.4 & $<0.5$ & 0.278 & 0.010 & 22.7 & 1.0 & -6.16 & -42.51 & & - & - \\
\hline 5 & 20.4 & 7.5 & ${ }_{1738}^{1738}$ & $\begin{array}{r}5.5 \\
38\end{array}$ & $\begin{array}{l}199.1 \\
1549\end{array}$ & 5.3 & 131.8 & 65.1 & 587.9 & 163.0 & 275.3 & 4.5 & $<0.5$ & 0.761 & 0.010 & 19.5 & 1.9 & -7.12 & -50.20 & & - & - \\
\hline $\begin{array}{l}6 \\
7\end{array}$ & $\begin{array}{l}21.8 \\
18.4\end{array}$ & $\begin{array}{l}7.7 \\
7.8\end{array}$ & $\begin{array}{l}1351 \\
1860\end{array}$ & $\begin{array}{l}3.8 \\
6.6\end{array}$ & $\begin{array}{l}154.9 \\
268.6\end{array}$ & $\begin{array}{l}5.4 \\
4.4\end{array}$ & $\begin{array}{l}102.5 \\
103.7\end{array}$ & $\begin{array}{l}44.2 \\
52.7\end{array}$ & $\begin{array}{l}376.4 \\
646.9\end{array}$ & $\begin{array}{l}146.9 \\
280.3\end{array}$ & 182.1 & 10.3 & $<0.5$ & 0.524 & 0.010 & $\begin{array}{l}21.6 \\
20.7\end{array}$ & $\begin{array}{c}5.2 \\
-0.5\end{array}$ & $\begin{array}{l}-6.69 \\
-654\end{array}$ & $\begin{array}{l}-47.75 \\
-44.9\end{array}$ & $\begin{array}{l}5.7 .7 \\
41.8\end{array}$ & $\begin{array}{l}-14.2 \\
-13.4\end{array}$ & $\begin{array}{l}100.8 \\
98.4\end{array}$ \\
\hline 8 & 19.8 & 7.8 & 1594 & 5.3 & 190.9 & 7.0 & 111.5 & 50.4 & 533.1 & 220.8 & 121.8 & 1.9 & $<0.5$ & 0.418 & 0.010 & 16.3 & 1.7 & -6.75 & -47.20 & $\begin{array}{lll}0 & 2.1 \\
0\end{array}$ & -13.8 & 97.3 \\
\hline 9 & 21.2 & 7.3 & 1907 & 7.9 & 202.9 & 4.8 & 134.1 & 78.5 & 681.9 & 251.5 & 145.5 & 22.2 & $<0.5$ & 0.841 & 0.010 & 22.0 & 1.6 & -6.90 & -47.92 & $2-$ & - & - \\
\hline 10 & 19.2 & 7.8 & 1332 & 4.3 & 164.8 & 4.4 & 85.1 & 41.9 & 405.7 & 140.5 & 140.1 & 12.9 & 0.6 & 0.589 & 0.010 & 19.4 & 4.8 & -6.86 & -47.57 & - & - & - \\
\hline 13 & 19.7 & 7.5 & 1319 & 5.3 & 170.2 & 4.2 & 83.0 & 40.8 & 459.1 & 160.6 & 147.6 & 12.8 & 0.6 & 0.562 & 0.010 & 18.9 & -1.0 & -6.84 & -47.80 & $0-$ & - & - \\
\hline $\begin{array}{l}14 \\
15\end{array}$ & $\begin{array}{l}17.6 \\
15.6\end{array}$ & $\begin{array}{l}8.6 \\
7.0\end{array}$ & $\begin{array}{l}1525 \\
437\end{array}$ & $\begin{array}{l}13.1 \\
9.2\end{array}$ & $\begin{array}{l}200.8 \\
43.1\end{array}$ & $\begin{array}{l}6.4 \\
3.5\end{array}$ & $\begin{array}{l}98.5 \\
28.1\end{array}$ & $\begin{array}{l}39.4 \\
12.2\end{array}$ & $\begin{array}{l}250.9 \\
162.5\end{array}$ & $\begin{array}{l}178.3 \\
340\end{array}$ & $\begin{array}{l}302.2 \\
28.2\end{array}$ & $\begin{array}{r}0.3 \\
182\end{array}$ & $\begin{array}{l}0.4 \\
0\end{array}$ & $\begin{array}{l}1.262 \\
0.238\end{array}$ & 0.013 & $\begin{array}{r}5.2 \\
13.2\end{array}$ & $\begin{array}{l}4.9 \\
-0.9\end{array}$ & $\begin{array}{l}-10.30 \\
-745\end{array}$ & $\begin{array}{l}-88.04 \\
-48.94\end{array}$ & & -8.7 & 90.6 \\
\hline $\begin{array}{l}15 \\
16\end{array}$ & $\begin{array}{l}15.6 \\
13.6\end{array}$ & $\begin{array}{l}7.0 \\
7.9\end{array}$ & $\begin{array}{l}437 \\
1070\end{array}$ & $\begin{array}{l}9.2 \\
5.3\end{array}$ & $\begin{array}{l}43.1 \\
125.2\end{array}$ & $\begin{array}{l}3.5 \\
5.1\end{array}$ & $\begin{array}{l}28.1 \\
77.0\end{array}$ & $\begin{array}{l}12.2 \\
25.5\end{array}$ & $\begin{array}{l}162.5 \\
503.1\end{array}$ & $\begin{array}{c}34.0 \\
109.2\end{array}$ & $\begin{array}{l}28.1 \\
12.6\end{array}$ & $\begin{array}{l}18.2 \\
0.2\end{array}$ & $\begin{array}{l}<0.5 \\
0.3\end{array}$ & $\begin{array}{l}0.238 \\
0.086\end{array}$ & $\begin{array}{l}0.010 \\
0.065\end{array}$ & $\begin{array}{l}13.3 \\
10.9\end{array}$ & $\begin{array}{l}-0.3 \\
-1.2\end{array}$ & $\begin{array}{l}-7.45 \\
-66\end{array}$ & $\begin{array}{l}-48.98 \\
-4688\end{array}$ & $\begin{array}{l}82.2 \\
8 \\
8.18\end{array}$ & $\begin{array}{l}-15.8 \\
\end{array}$ & $\begin{array}{c}96.0 \\
10.17\end{array}$ \\
\hline 17 & 19.4 & 7.7 & $\begin{array}{l}1180 \\
1189\end{array}$ & $\begin{array}{l}.5 \\
8.3\end{array}$ & $\begin{array}{l}121.9 \\
121.9\end{array}$ & $\begin{array}{l}.1 \\
2.6\end{array}$ & 85.0 & 30.1 & $\begin{array}{l}003.1 \\
303.4\end{array}$ & $\begin{array}{l}109.2 \\
140.7\end{array}$ & $\begin{array}{l}121.6 \\
71.4\end{array}$ & $\begin{array}{l}0.2 \\
35.5\end{array}$ & $\begin{array}{l}0.3 \\
0.4\end{array}$ & $\begin{array}{l}0.826 \\
0.651\end{array}$ & $\begin{array}{l}0.0650 \\
0.010\end{array}$ & $\begin{array}{l}10.9 \\
26.8\end{array}$ & $\begin{array}{c}-1.2 \\
6.6\end{array}$ & $\begin{array}{l}-6.60 \\
-7.15\end{array}$ & $\begin{array}{l}-46.88 \\
-48.88\end{array}$ & $8-8$ & -12.6 & 101.7 \\
\hline 18 & 18.6 & 7.7 & 1299 & 10.8 & 136.5 & 2.5 & 102.8 & 35.5 & 445.3 & 151.3 & 100.2 & 8.2 & 0.6 & 0.682 & 0.010 & 19.7 & 0.6 & -8.25 & -60.33 & 32.3 & -14.2 & 103.5 \\
\hline 19 & 17.3 & 7.8 & 854 & 11.8 & 197.4 & 7.2 & 117.8 & 35.5 & 795.7 & 95.6 & 31.0 & 10.1 & $<0.5$ & 0.457 & 0.010 & 9.0 & 2.8 & -7.54 & -51.83 & $\begin{array}{l}31.9 \\
\end{array}$ & -13.6 & 107.4 \\
\hline 20 & 18.9 & 7.9 & 637 & 10.8 & 71.3 & 2.7 & & 13.0 & 288.2 & 50.5 & 21.6 & 12.3 & $<0.5$ & & 0.010 & 10.2 & -1.4 & -7.54 & -51.35 & 51.8 & -12.5 & 108.8 \\
\hline 24 & 20.3 & 7.4 & 2350 & 2.8 & 307.8 & 2.4 & 132.1 & $\begin{array}{l}74.8 \\
.7 .8\end{array}$ & 447.6 & 317.6 & 409.4 & 0.2 & 0.8 & 0.030 & 0.180 & 16.3 & 2.3 & -8.82 & -72.73 & 35.5 & -14.7 & 102.2 \\
\hline 25 & $\begin{array}{l}18.4 \\
\end{array}$ & 7.4 & 664 & $\begin{array}{l}7.3 \\
\end{array}$ & $\begin{array}{r}74.9 \\
\end{array}$ & 2.9 & $\begin{array}{l}42.3 \\
757.3\end{array}$ & 12.5 & $\begin{array}{l}214.0 \\
\end{array}$ & $\begin{array}{l}86.2 \\
1185\end{array}$ & $\begin{array}{r}28.5 \\
1118\end{array}$ & 2.1 & $<0.5$ & 0.051 & 0.010 & 22.0 & -2.8 & $\begin{array}{l}-7.92 \\
767\end{array}$ & -56.76 & $6<0.5$ & -13.3 & $\begin{array}{l}98.9 \\
100\end{array}$ \\
\hline $\begin{array}{l}26 \\
27\end{array}$ & $\begin{array}{l}15.8 \\
166\end{array}$ & $\begin{array}{l}7.6 \\
74\end{array}$ & $\begin{array}{l}1655 \\
7665\end{array}$ & $\begin{array}{l}5.2 \\
5.8\end{array}$ & $\begin{array}{l}253.3 \\
927\end{array}$ & $\begin{array}{l}4.8 \\
1.3\end{array}$ & $\begin{array}{l}75.7 \\
571\end{array}$ & $\begin{array}{l}24.7 \\
16.7\end{array}$ & $\begin{array}{l}421.1 \\
2866\end{array}$ & $\begin{array}{l}178.5 \\
1040\end{array}$ & $\begin{array}{l}118.6 \\
294\end{array}$ & $\begin{array}{l}28.6 \\
02\end{array}$ & $\begin{array}{l}2.2 \\
06\end{array}$ & $\begin{array}{l}0.110 \\
0.086\end{array}$ & $\begin{array}{l}0.010 \\
0.019\end{array}$ & $\begin{array}{l}19.4 \\
189\end{array}$ & $\begin{array}{c}7.3 \\
-21\end{array}$ & $\begin{array}{l}-7.67 \\
-762\end{array}$ & $\begin{array}{l}-54.40 \\
-516\end{array}$ & $\begin{array}{ll}0 & 1.0 \\
5 & <09\end{array}$ & $\begin{array}{l}-13.3 \\
-148\end{array}$ & $\begin{array}{l}102.6 \\
95.7\end{array}$ \\
\hline 28 & $\begin{array}{l}16.6 \\
14.4\end{array}$ & $\begin{array}{l}1.4 \\
7.7\end{array}$ & 616 & $\begin{array}{l}.8 .8 \\
6.1\end{array}$ & $\begin{array}{l}9.2 .7 \\
81.9\end{array}$ & $\begin{array}{l}1.3 \\
2.0\end{array}$ & $\begin{array}{r}77.1 \\
37.9\end{array}$ & $\begin{array}{l}16.1 \\
12.7\end{array}$ & $\begin{array}{l}286.6 \\
293.0\end{array}$ & $\begin{array}{l}104.0 \\
75.6\end{array}$ & $\begin{array}{l}29.4 \\
27.7\end{array}$ & $\begin{array}{l}.2 \\
1.6\end{array}$ & $\begin{array}{l}.0 .6 \\
<0.5\end{array}$ & $\begin{array}{l}0.006 \\
0.058\end{array}$ & $\begin{array}{l}0.1919 \\
0.023\end{array}$ & $\begin{array}{l}18.9 \\
13.3\end{array}$ & $\begin{array}{l}-2.1 \\
-9.3\end{array}$ & $\begin{array}{l}-7.62 \\
-7.51\end{array}$ & $\begin{array}{l}-51.65 \\
-51.60\end{array}$ & $\begin{array}{l}0<0.9 \\
0 \quad 2.2\end{array}$ & $\begin{array}{l}-14.8 \\
-13.6\end{array}$ & $\begin{array}{l}90.7 \\
101.3\end{array}$ \\
\hline 29 & 17.9 & 7.6 & 508 & 5.2 & 50.4 & 2.3 & 37.7 & 13.0 & 248.9 & 41.8 & 13.8 & 5.6 & $<0.5$ & 0.022 & 0.010 & 10.9 & -6.5 & -7.15 & -49.04 & 41.5 & -15.3 & 107.3 \\
\hline 32 & 17.9 & 7.1 & 967 & 5.0 & 115.8 & 2.4 & 46.0 & 25.9 & 200.5 & 118.0 & 150.9 & 0.5 & $<0.5$ & 0.039 & 0.026 & 20.6 & -3.3 & -6.29 & -47.64 & 41.8 & -15.5 & 101.2 \\
\hline 33 & 16.1 & 7.7 & 7480 & 5.8 & 747.6 & 185.6 & 374.3 & 247.7 & 1238.7 & 1584.4 & 574.8 & 15.8 & $<0.5$ & 0.002 & 0.078 & 18.7 & -0.7 & -5.83 & -47.67 & 72.2 & -10.2 & 106.1 \\
\hline
\end{tabular}


Table 2 summarizes the average values of physicochemical parameters for groundwater from 1981 and 2014. As shown, groundwater samples from 2014 had higher $\mathrm{Ca}^{2+}, \mathrm{Mg}^{2+}, \mathrm{Na}^{+}, \mathrm{K}^{+}, \mathrm{Cl}^{-}$, $\mathrm{HCO}_{3}^{-}$, and $\mathrm{NO}_{3}^{-}$concentrations, and hence higher EC values, than groundwater samples from 1981. The concentrations for $\mathrm{SO}_{4}^{2-}$ remained practically unchanged. The hydrochemical difference was confirmed by a $t$-test (Table 2); among the physicochemical parameters measured, only $\mathrm{Ca}^{2+}$ and $\mathrm{HCO}_{3}^{-}$were significantly different $(p$ value $<0.05)$ between the two sampling campaigns, while the rest did not show any significant difference. From this is can be concluded that there exists an increasing trend between 1981 and 2014, however, it is not significant for most physicochemical parameters.

Table 2. Average, standard deviation $( \pm 1 \sigma)$ and $p$-values of various physicochemical parameters for the Tecate aquifer.

\begin{tabular}{|c|c|c|c|c|c|c|}
\hline Year & Measures & $\mathrm{CE}(\mu \mathrm{S} / \mathrm{cm})$ & $\mathrm{pH}$ & $\mathrm{Ca}\left(\mathrm{mg} \mathrm{L}^{-1}\right)$ & $\operatorname{Mg}\left(\mathrm{mg} \mathrm{L}^{-1}\right)$ & $\mathrm{Na}\left(\mathrm{mg} \mathrm{L}^{-1}\right)$ \\
\hline 1981 & Avg. \pm St.dev & $1122 \pm 739$ & $8.1 \pm 0.4$ & $51.3 \pm 16.6$ & $40.6 \pm 33.2$ & $138.3 \pm 139.5$ \\
\hline 2014 & $\begin{array}{c}\text { Avg. } \pm \text { St.dev. } \\
p \text {-value }\end{array}$ & $\begin{array}{c}1622 \pm 1642 \\
0.001^{*} \\
\mathbf{K}\left(\mathbf{m g ~ L}^{-1}\right) \\
\end{array}$ & $\begin{array}{c}7.6 \pm 0.3 \\
0.001^{*} \\
\mathrm{Cl}\left(\mathrm{mg} \mathrm{L}^{-\mathbf{1}}\right) \\
\end{array}$ & $\begin{array}{c}103.0 \pm 79.4 \\
0.021 * \\
\mathbf{H C O}_{3}\left(\mathbf{m g ~ L}^{-1}\right)\end{array}$ & $\begin{array}{c}49.3 \pm 56.7 \\
0.584 \\
\mathbf{S O}_{\mathbf{4}}\left(\mathbf{m g ~ L}^{-\mathbf{1}}\right)\end{array}$ & $\begin{array}{c}193.0 \pm 163.3 \\
0.269 \\
\mathrm{NO}_{\mathbf{3}}\left(\mathbf{m g ~ L}^{-\mathbf{1}}\right)\end{array}$ \\
\hline 1981 & Avg. \pm St.dev & $4.3 \pm 2.1$ & $175.9 \pm 184.7$ & $207.6 \pm 45.6$ & $159.0 \pm 205.7$ & $7.2 \pm 6.3$ \\
\hline 2014 & $\begin{array}{c}\text { Avg. } \pm \text { St.dev. } \\
p \text {-value }\end{array}$ & $\begin{array}{c}15.2 \pm 45.5 \\
0.346\end{array}$ & $\begin{array}{c}232.5 \pm 367.1 \\
0.577\end{array}$ & $\begin{array}{c}472.7 \pm 261.6 \\
0.001 *\end{array}$ & $\begin{array}{c}151.5 \pm 161.6 \\
0.887\end{array}$ & $\begin{array}{c}8.8 \pm 11.1 \\
0.640\end{array}$ \\
\hline
\end{tabular}

With $\sim 52 \%$ of equivalent anion chemistry, $\mathrm{HCO}_{3}^{-}$is the dominant species among anions. Weathering of alumino-silicate rocks and the contribution of $\mathrm{CO}_{2}$ gas dissolution are supposed to be the primary sources of $\mathrm{HCO}_{3}^{-}$. Biodegradation of organic matter and root respiration increases $\mathrm{CO}_{2}$ pressure in soils. The infiltrating water absorbs $\mathrm{CO}_{2}$ by generating $\mathrm{HCO}_{3}$. Chloride makes up roughly $31 \%$ of anion chemistry. Generally, $\mathrm{Cl}^{-}$has its origin in atmospheric precipitation or mixing with seawater. The $\mathrm{Na} / \mathrm{Cl}$ molar ratio ranges from 0.7 to 3.2 (average 1.7). Halite dissolution would produce a $\mathrm{Na} / \mathrm{Cl}$ ratio equal to 1.0 , and seawater close to $0.85 . \mathrm{Na} / \mathrm{Cl}$ ratios decrease with increasing salinity, which is an indication that the evaporation effect is not a dominant process. Concentration mainly by evaporation process would leave the $\mathrm{Na} / \mathrm{Cl}$ ratio unchanged. Rather, the higher $\mathrm{Na} / \mathrm{Cl}$ ratio suggests silicate weathering and/or anthropogenic sources such as agriculture runoff or waste as the main processes. Sulfate concentration is in the order of $16 \%$ of total anion chemistry. Lower concentrations in the study area were most probably due to rock weathering, while higher concentrations above $60 \mathrm{mg} \mathrm{L}^{-1}$ were attributable to the urban area indicating anthropogenic sources like industrial effluents. The significant correlation between $\mathrm{SO}_{4}{ }^{2-}$ and $\mathrm{Cl}^{-}$concentrations $\left(\mathrm{r}^{2}=0.79 ; \mathrm{t}=0.000\right)$ confirms this assumption. Finally, $\mathrm{NO}_{3}^{-}$concentrations in groundwater constituted $\sim 2 \%$ of total anionic concentrations. Nitrate generally derives from agricultural fertilizers, human and animal excreta, biological fixation and nitrification processes, and atmospheric precipitation [39-41]. Fluoride concentration in groundwater (up to $2.2 \mathrm{mg} \mathrm{L}^{-1}$ ) exceeds the national drinking water standard $\left(1.5 \mathrm{mg} \mathrm{L}^{-1}\right)$ in only one sample. In volcanic environments, $\mathrm{F}^{-}$usually originates from weathering of fluoride-bearing rock forming minerals like fluorite, biotite and muscovite [39,42].

Alkali metals $\mathrm{Na}^{+}\left(43-748 \mathrm{mg} \mathrm{L}^{-1}\right)$ and $\mathrm{K}^{+}\left(1.3-186 \mathrm{mg} \mathrm{L}^{-1}\right)$ account together for about $48 \%$ of the total cations equivalency, respectively. The origin of these ions in the studied waters is primarily weathering of rock-forming silicates such as plagioclase, andesine and orthoclase, which are part of a granodiorite, diorite and tonalite rock matrix. Alkaline earth metals $\mathrm{Ca}^{2+}\left(28.1-374.3 \mathrm{mg} \mathrm{L}^{-1}\right)$ and $\mathrm{Mg}^{2+}\left(12.2-247.7 \mathrm{mg} \mathrm{L}^{-1}\right)$ account for $31 \%$ and $21 \%$ of total cations, respectively. The presence of these ions may indicate weathering of minerals such hornblende, olivine, pyroxene, plagioclase and, to a smaller extent, calcite dissolution. Iron $\left(<0.18 \mathrm{mg} \mathrm{L}^{-1}\right)$ is detected in only $27 \%$ of samples (detection limit $=0.02 \mathrm{mg} \mathrm{L}^{-1}$ ). This suggests association of $\mathrm{Ca}^{2+}$ and $\mathrm{Mg}^{2+}$ with plagioclases rather than with mafic minerals. 


\subsection{Hydrochemical Clusters}

In order to understand local chemical trends, it is necessary to classify samples according to their chemical similarity and geographical correspondence. A hierarchical cluster analysis classifies samples into 3 groups: Group 1 is located inside the triangle with vertices Tecate, La Rumorosa and El Hongo; Group 2 is located in Tecate City and the towns of La Rumorosa and El Hongo; and Group 3 is located towards the El Carrizo dam and Nueva Colonia Hindú locality south and southwest of Tecate City (Figure 1). Trilinear diagrams for cations and anions show the chemical differences between the three groups (Figure 2).
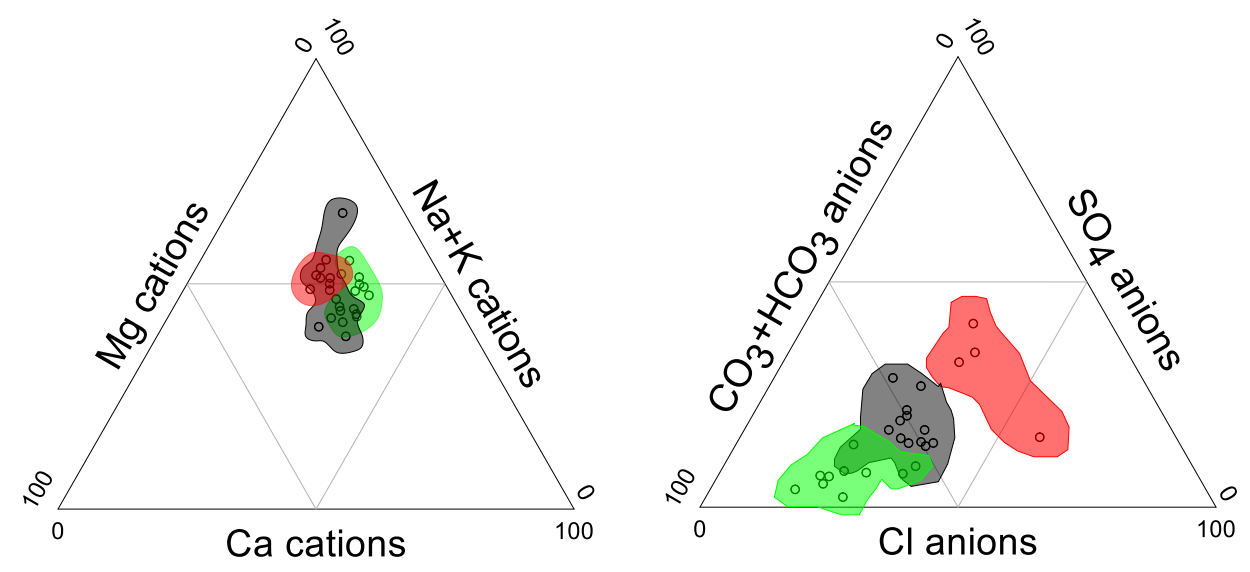

Figure 2. Trilinear diagrams for cations and anions indicating the HCA statistical groups: Group 1, in green, stands for samples located in the triangle of Tecate-La Rumorosa-El Hongo. Group 2, in grey, represent samples in Tecate, La Rumorosa and El Hongo. Group 3, in red, considers samples south and southeast of Tecate.

While the differences in the cation concentrations are relatively small, for the anions there is a clear increasing trend of relative $\mathrm{Cl}^{-}$and $\mathrm{SO}_{4}^{2-}$ content from Group 1 to Group 3, while lowering the relative $\mathrm{HCO}_{3}^{-}$contribution. Group 1 is a mixed- $-\mathrm{HCO}_{3}$ to $\mathrm{Na}-\mathrm{HCO}_{3}$ water type and Group 2 is a mixed- $\mathrm{HCO}_{3}$ to $\mathrm{Na}-\mathrm{HCO}_{3}$ to mixed-mixed type. There is slight overlapping between Group 1 and 2 . Finally, Group 3 is a mixed-mixed to Na-mixed type. This sequence of groups displays a relatively uniform geochemical evolution suggesting water-rock interaction, i.e., weathering of mostly acid silicate rocks among other processes.

\subsection{Statistical Variation of Selected Variables}

The classification of samples into groundwater groups also reveals a more detailed picture about groundwater evolution, when looking to the comparison of statistics of selected variables in form of boxplots (Figure 3). Group 1 has a lower mineralization (average $695 \mu \mathrm{S} \mathrm{cm}{ }^{-1}$ ) and temperature $\left(17.0^{\circ} \mathrm{C}\right)$ in comparison to the other groups, reflecting a low circulation and recent recharge and pristine conditions in the hilly central to northeastern area. Group 2, located mainly in Tecate Valley shows a general trend of increased temperature $\left(19.6^{\circ} \mathrm{C}\right)$ and mineralization $\left(1424 \mu \mathrm{S} \mathrm{cm}{ }^{-1}\right)$, suggesting more evolved groundwater. The concentration of $\mathrm{SO}_{4}^{2-}\left(131.0 \mathrm{mg} \mathrm{L}^{-1}\right)$ and $\mathrm{NO}_{3}^{-}\left(11.9 \mathrm{mg} \mathrm{L}^{-1}\right)$ is almost five times and two times higher than the first group, respectively, which indicates a significant human impact on groundwater resources. The distribution of nitrate concentrations shows that high values are found in both urban (Tecate city) and rural (around El Hongo) areas. The elevated concentrations in urban Tecate point to infiltration of treated wastewater from the Tecate river and leaching from waste disposal. In the rural area, the high concentrations indicate agricultural activities involving nitrogen components such as fertilizers or organic byproducts, septic tanks and livestock manure near El Hongo. Finally, Group 3 integrates samples located in the southwestern study area with a temperature of 
$18.0^{\circ} \mathrm{C}$ and largely variable concentrations, which is generally higher and more heterogeneous than previous groups. Similarly, this group shows an important deterioration in water quality regarding $\mathrm{Cl}^{-}, \mathrm{NO}_{3}^{-}$and $\mathrm{SO}_{4}^{2-}$ concentrations.
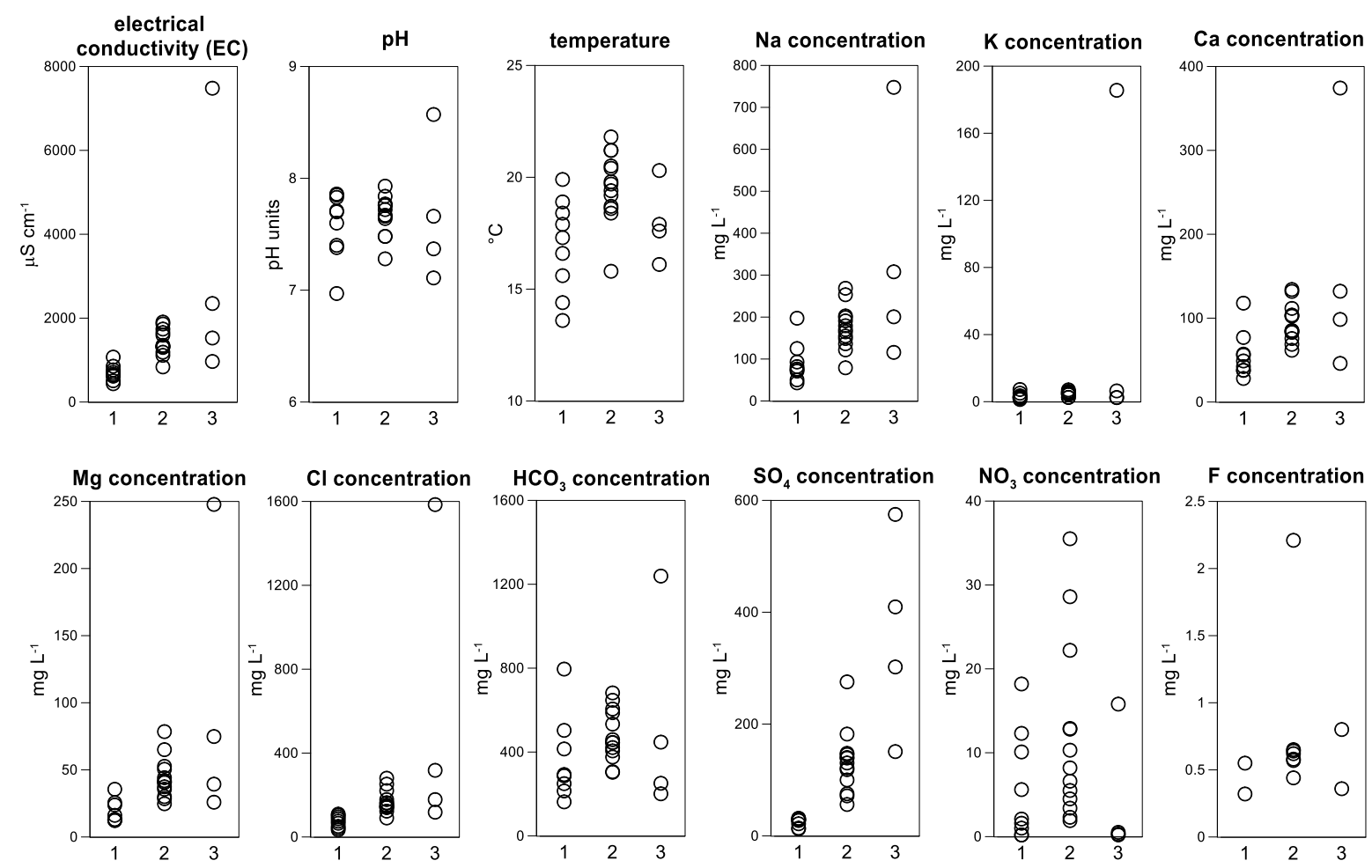

Figure 3. Ranges of selected variables, according to the 3 groups.

\subsection{Identification of Hydrogeochemical Processes}

Scatter diagrams show a strong correlation between $\mathrm{Cl}^{-}$and $\mathrm{Na}^{+}$, and $\mathrm{Na}^{+}$excess in relation to $\mathrm{Cl}^{-}$(Figure 4a). This reflects that besides halite dissolution, other processes such as plagioclase weathering (mainly albite) and/or $\mathrm{Na}-\mathrm{Ca}$ ion exchange could be taking place. Another alternative would be the effect of sodium-rich wastewater disposal from households [43]. This could explain why the $\mathrm{Na} / \mathrm{Cl}$ ratio is higher in urban Group 2 and 3 than in rural Group 1. The plot $(\mathrm{Ca}+\mathrm{Mg})$ versus $\left(\mathrm{HCO}_{3}+\mathrm{SO}_{4}\right)$ in Figure $4 \mathrm{~b}$ shows that all points fall below the 1:1 equiline, which suggests that the $\mathrm{Ca}$ and $\mathrm{Mg}$ chemistry is largely explained by silicate weathering processes. In order to evaluate this assumption, a plot of $\mathrm{Ca} / \mathrm{Na}$ vs. $\mathrm{HCO}_{3} / \mathrm{Na}$ is given (Figure 4c). It appears from this molar relationship that groundwater samples are all close to typical values for silicate weathering tending to evaporate weathering. Therefore, other processes such as carbonate dissolution are of little importance for groundwater acquiring ion concentrations during water-rock interaction. Ion exchange processes can be evaluated using the relationship between $\left(\mathrm{Ca}+\mathrm{Mg}-\mathrm{HCO}_{3}-\mathrm{SO}_{4}\right)$ and $(\mathrm{Na}+\mathrm{K}-\mathrm{Cl})$. If ion exchange plays a predominant role in the system, then the values should fall along a line with a slope of -1 . This is not the case for our study area (Figure $4 \mathrm{~d}$ ). The data show that the average slope is -0.80 $\left(\mathrm{r}^{2}=0.84\right)$, which suggests that cation exchange of $\mathrm{Ca}$ and $\mathrm{Mg}$ for $\mathrm{Na}$ is important, but other processes such as albite weathering are also present. The slopes of each group are relatively similar to each other and range from -0.72 to -0.83 . 

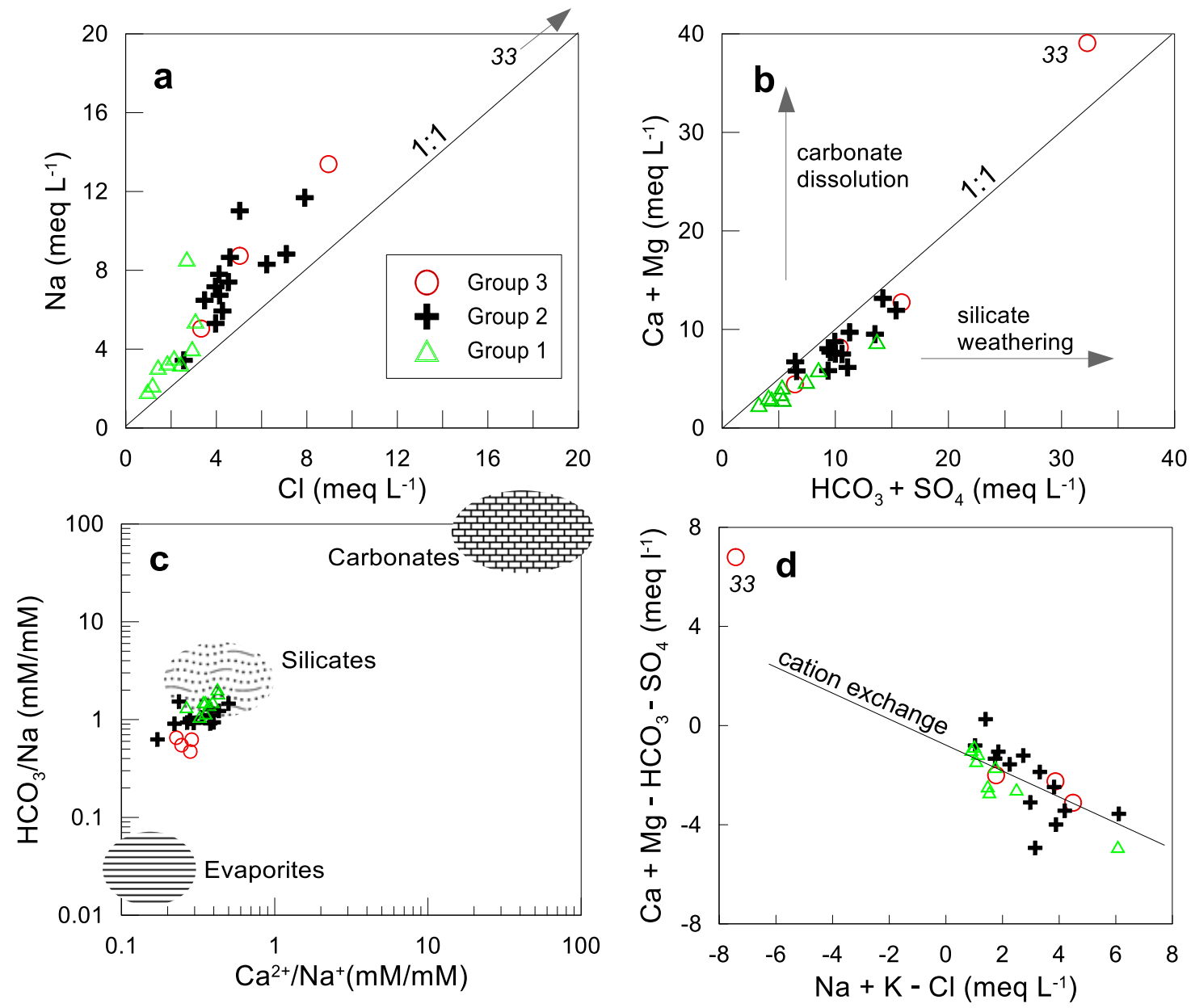

Figure 4. Scatterplots used to assess hydrogeochemical processes: (a) $\mathrm{Na}$ vs. $\mathrm{Cl}$; (b) $\left(\mathrm{HCO}_{3}+\mathrm{SO}_{4}\right)$ vs. $(\mathrm{Ca}+\mathrm{Mg}) ;\left(\right.$ c) $\mathrm{Ca} / \mathrm{Na}$ vs. $\mathrm{HCO}_{3} / \mathrm{Na} ;\left(\right.$ d) $\mathrm{Na}+\mathrm{K}-\mathrm{Cl}$ vs. $\mathrm{Ca}+\mathrm{Mg}-\mathrm{HCO}_{3}-\mathrm{SO}_{4}$.

Water infiltrated recently has low concentrations of chemical constituents. As water moves along the flow path and the travel time is longer, more ions are acquired along the flow path. In our case, Group 1 is the least mineralized water and Groups 2 and 3 show increased TDS (Figure 5a). $\mathrm{EC}$ and $\mathrm{Cl}^{-}$concentrations increase exponentially along with the flow (Figure 5b). Group 1 water is associated with recent recharge and short transit times (local flow), while group 2 and 3 with greater distances and longer residence times. Group 1 also shows lower temperatures, $\mathrm{Cl}^{-}$and $\mathrm{SO}_{4}^{2-}$ concentrations compared to Group 2 (Figure $5 \mathrm{~b}, \mathrm{~d}$ ). Group 2 exhibits the largest variation and highest values of $\mathrm{NO}_{3}^{-}$among all groups. Group 3 displays the highest $\mathrm{SO}_{4}^{2-}$ values but the lowest $\mathrm{NO}_{3}^{-}$ concentrations (Figure $5 c$,d). In general, salinity increases with temperature, i.e., a trend from a low temperature/salinity in Group 1 to elevated temperature/salinity in Group 3 (Figure 5e). Given the fact that the depth of the water table ranges from 2.1 to $15.3 \mathrm{~m}$ [2], the aquifer system is prone to local recharge even of poor water quality. 

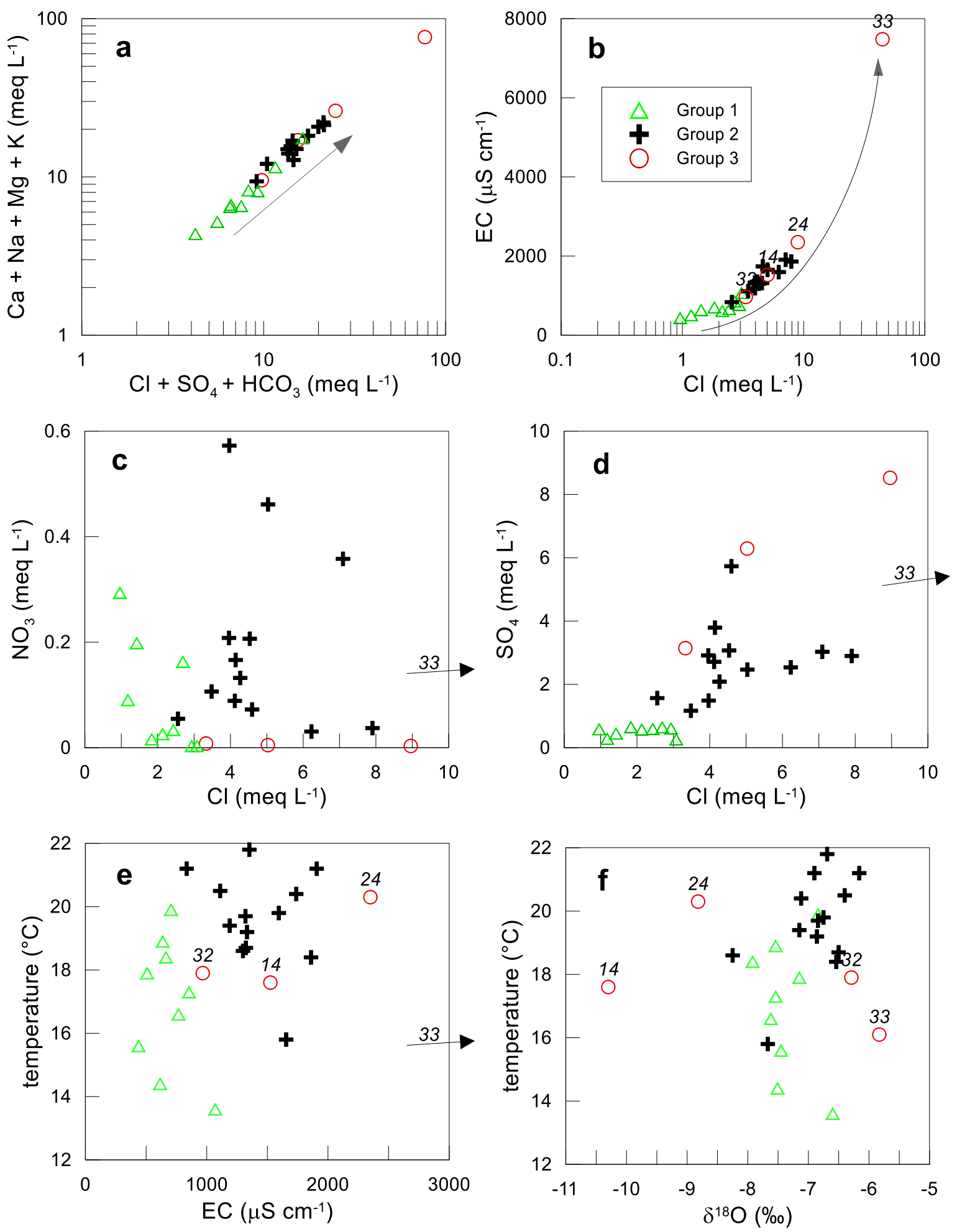

Figure 5. Scatter plots for evaluation of hydrogeochemical processes: (a) $(\mathrm{Ca}+\mathrm{Na}+\mathrm{Mg}+\mathrm{K})$ vs. $(\mathrm{Cl}+$ $\mathrm{SO}_{4}+\mathrm{HCO}_{3}$ ); (b) EC vs. $\mathrm{Cl}$; (c) $\mathrm{NO}_{3}$ vs. $\mathrm{Cl}$; (d) $\mathrm{SO}_{4}$ vs. $\mathrm{Cl}$; (e) salinity vs. temperature; (f) oxygen-18 vs. temperature.

\subsection{Geochemical Trends}

Stability diagrams of $\mathrm{Ca}-\mathrm{Al}, \mathrm{Na}-\mathrm{Al}, \mathrm{Mg}-\mathrm{Al}$, and $\mathrm{K}-\mathrm{Al}$ silicate phases are useful to study the occurrence of incongruent dissolution (e.g., [41,44]). Figure 6a shows that most water samples are 
found in the limit between the stability field of kaolinite and Ca-Montmorillonite, and some samples are located in the Ca-montmorillonite, Na-montmorillonite and $\mathrm{Mg}$-smectite fields, respectively. Thus, albite, anorthite, gibbsite, muscovite and orthoclase (microcline) would be unstable, facilitating their dissolution and contribution to the presence of $\mathrm{Na}^{+}, \mathrm{Ca}^{2+}$ and $\mathrm{Mg}^{2+}$ in groundwater. Based on these data, the reactions involving the incongruent dissolution of albite to kaolinite or Na-montmorillonite, and anorthite to kaolinite or Ca-montmorillonite, and orthoclase/muscovite to kaolinite would be mostly favored in this aquifer. This means that infiltrating water enriched with soil $\mathrm{CO}_{2}$ reacts with silicate minerals from the host rocks, albite, anorthite and orthoclase, leaching out $\mathrm{Na}^{+}, \mathrm{K}^{+}, \mathrm{Mg}^{2+}$ and $\mathrm{HCO}_{3}^{-}$, and converting in kaolinite, montmorillonite and silica. Besides, other processes occur such as ion exchange on clayey surfaces that set free $\mathrm{Na}^{+}$.
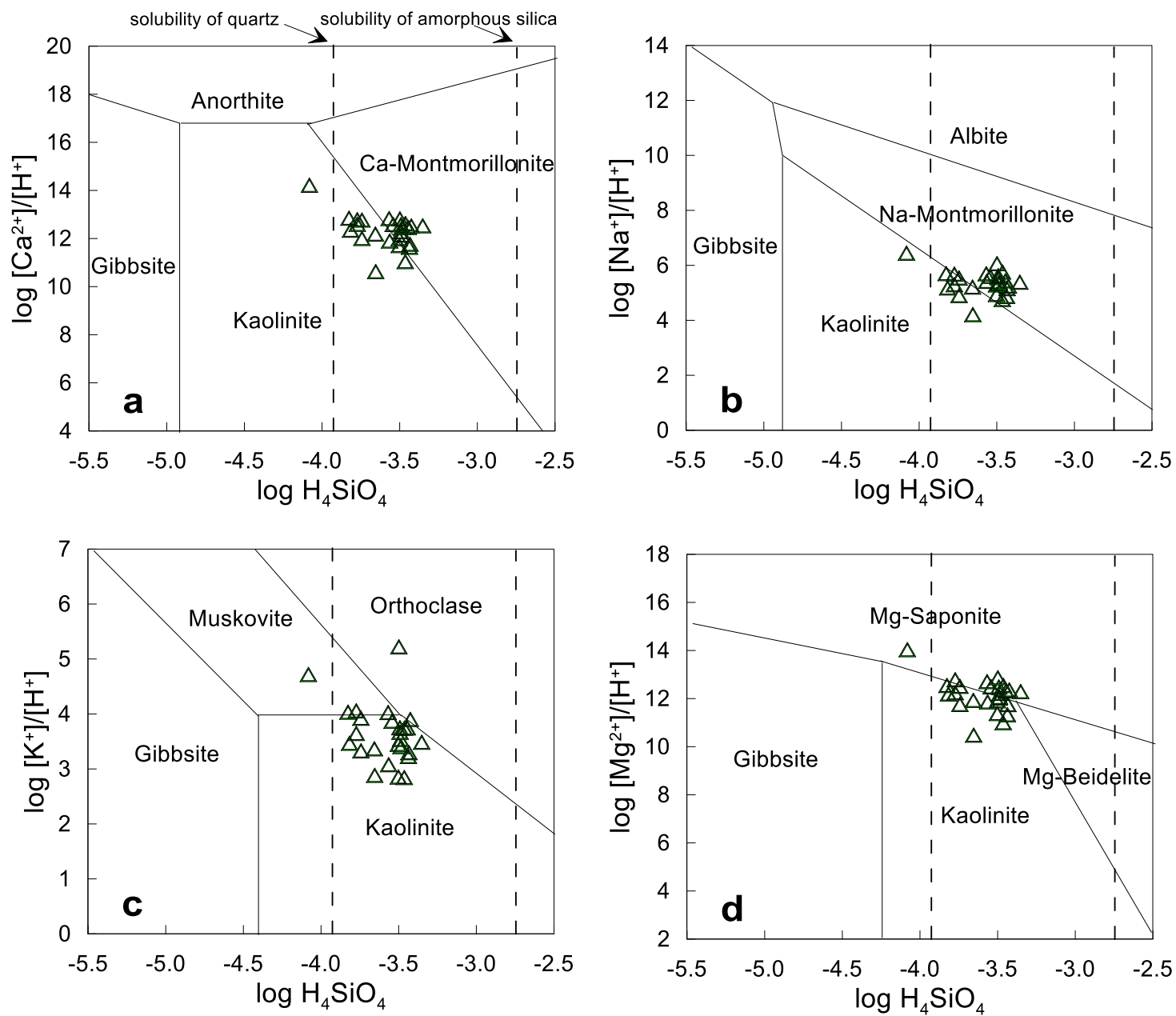

Figure 6. Stability of (a) Ca-Al silicate phases; (b) Na-Al silicate phases; (c) K-Al silicate phases; and (d) $\mathrm{Mg}$-Al phases in aqueous solution at $25^{\circ} \mathrm{C}$ and $1 \mathrm{~atm}$ pressure with groundwater data of the study area.

Saturation indexes are valuable in predicting the presence and trend of reactive minerals in groundwater. The results show that the samples of all groups are under-saturated with respect to halite, and silica, signaling their dissolution from mineral phases (Figure 7). There is a trend of water towards saturation with respect to albite, meaning that this mineral tends to dissolve in water under the given conditions. The calcite saturation index shows a trend from under-saturation to over-saturation. The large variation of SIs for calcite is remarkable. The over-saturation is probably due to the dissolution of Ca-containing minerals such as anorthite. The figures for Ca-montmorillonite, quartz, biotite and kaolinite exhibit over-saturation, which suggests a trend to precipitation of these mineral phases. This is congruent with the most common minerals that compose the rocks in the study 
area and with stability diagrams (Figure 6). The $\mathrm{P}_{\mathrm{CO} 2}$ values $\left(10^{-3.3}-10^{-1.5}\right)$ are in the order of soils where bacterial oxidation of vegetation and respiration of $\mathrm{CO}_{2}$ in the root zone occurs.
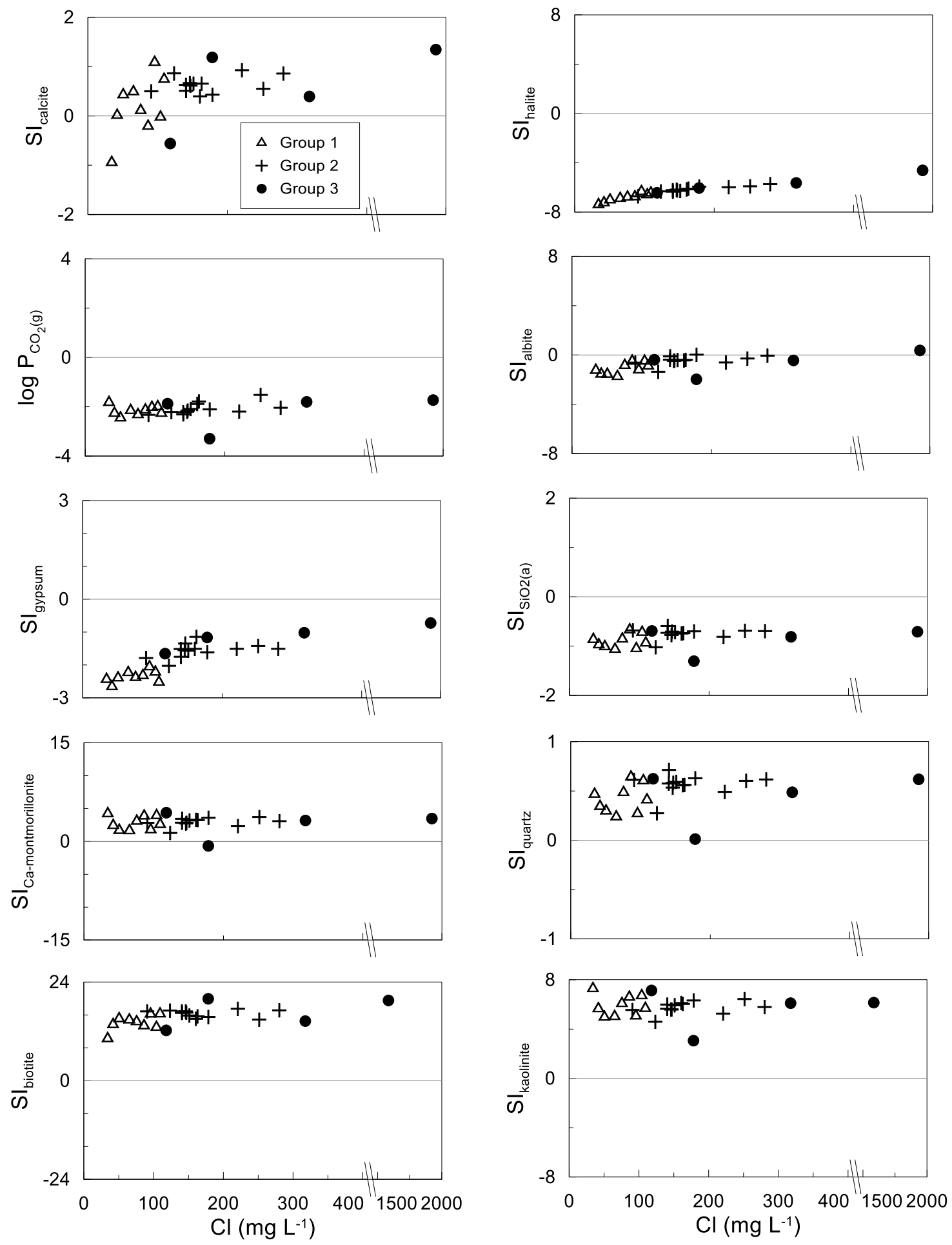

Figure 7. Saturation indexes versus chloride concentrations of relevant reacting mineral phases.

\subsection{Stable Isotopes}

Stable water isotopes are a powerful means to study the water cycle. Specifically, the origin and exchange processes may be studied using isotope ratios and deuterium excess. ${ }^{18} \mathrm{O}$ and ${ }^{2} \mathrm{H}$ ratios 
vary from -10.3 to $-6.2 \%$ and from -88.0 to $-42.5 \%$, respectively (Table 1 ). A comparison of the isotope ratios in groundwater from the study site with those of the global meteoric water line [45] and the regional meteoric water line (RMWL) (International Atomic Energy Agency-Global Network of Isotopes in Precipitation (IAEA-GNIP) station for Chihuahua), suggests that groundwater sampled in this study is of meteoric origin and has undergone varying degrees of evaporation (Figure 8a). Most samples are located below the present-day RMWL and define a linear trend described by the relationship $\delta^{2} \mathrm{H}=4.25 \delta^{18} \mathrm{O}-18.71$ (evaporation trend, ET), which is indicative of evaporation during recharge. The evaporation trend has an intercept on the meteoric water line at $-7.65 \%$, close to the composition of modern precipitation in this area, indicating that this groundwater is derived from modern precipitation that is exposed to evaporation during its passage through the soil and unsaturated zone to reach the water table. The range of values from $\delta^{2} \mathrm{H}$ and $\delta^{18} \mathrm{O}$ is similar to those reported in the areas of La Mision and Valle de Guadalupe [46,47] which are near (ca. $100 \mathrm{~km}$ ) to the study area and adjacent to the Pacific Ocean.
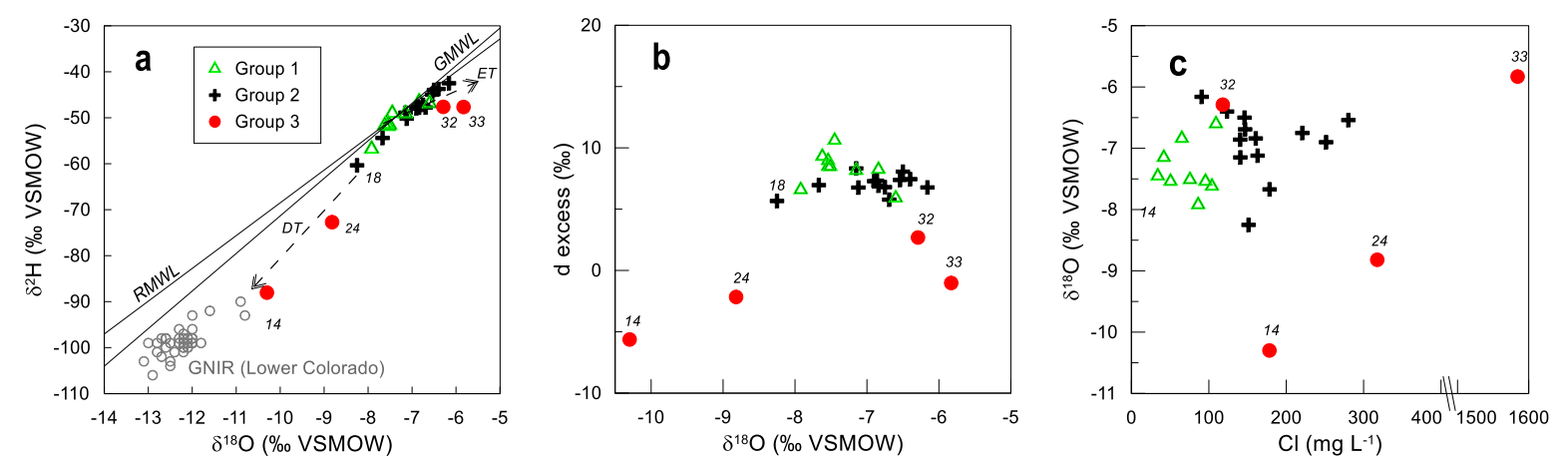

Figure 8. (a) $\delta^{18} \mathrm{O}$ and $\delta^{2} \mathrm{H}$ in groundwater from the study area; (b) $\delta^{18} \mathrm{O}$ and ${ }^{2} \mathrm{H}$ excess; (c) $\delta^{18} \mathrm{O}$ compared to chloride concentration. Labels indicate selected sample numbers. ET means evaporation trend. DT means dilution trend.

Another subset of samples shows a trend to more depleted values in comparison to RMWL (No. 14, 24, 18, 25). Sample No. 14 is close to the Las Auras dam, and sample No. 24 is close to the El Carrizo dam. This is indicative that these waters are modified due to a mixture of water from surface water bodies which are supplied by water with different meteoric origin, such as that derived from the Colorado River-Tijuana aqueduct that supplies these dams. A comparison with synoptic data retrieved from the Global Network of Isotopes for Rivers (GNIR-IAEA) for the lower Colorado River basin evidences this mixture of water (DT; Figure 8a), being sample No. 14 closest to the isotopic composition of river water.

Deuterium excess varies mostly between $5 \%$ and $10 \%$ (Figure $8 \mathrm{~b}$ ). Low deuterium excess values $(<10 \%)$ may be caused by post-condensation evaporative effects, whereby falling rain drops in warm regions having low relative humidity undergo slight re-evaporation before hitting the ground, a feature generally seen only in summertime $[48,49]$. From these observations, it can be concluded that there are at least two recharge mechanisms: (i) recharge from mountainous areas, which enters and travels through the fractured/porous aquifer as horizontal flow; (ii) vertical recharge to the aquifer as a product of rainwater or anthropogenic activities at the surface. Not only are $\mathrm{Cl}^{-}$concentrations and chemistry quite different between Groups 1 and 2, but also their isotopic signature. Most points of Group 1 have $-7.5 \%$ as $\delta^{18} \mathrm{O}$, whereas most points of Group 2 have $-6.5 /-7 \%$ (Figure $8 \mathrm{c}$ ). In general, the stable water isotopes are not strongly affected by evaporation as expected from the dry climate of the region. This can be explained by the rapid infiltration process in the mountainous recharge areas via fractures. With regard to Group 1 , the $\delta^{18} \mathrm{O}$ signature increases with cumulative salinity, and for Groups 2 and 3, no clear trend is observable (Figure 5e). This is in part due to the effect of water from the aqueduct, at least in several samples. 


\subsection{Groundwater Age}

Groundwater age represents a direct indicator of the transit time of water through a catchment and is therefore a useful parameter for describing catchment processes and movement of contaminants. The results for ${ }^{3} \mathrm{H}$ and ${ }^{14} \mathrm{C}$ are presented in Table 1. Tritium activities range from $<0.5$ to $7.4 \mathrm{TU}$. The IAEA-GNIP station for Chihuahua ( $\sim 1100 \mathrm{~km}$ SE from Tecate) reports that the activity of ${ }^{3} \mathrm{H}$ has been decreasing since the 1960s and its current level is about 2 to 3 TU [21]. Radiocarbon values vary from 90.6 to $108.8 \mathrm{pMC}$.

The local input function of ${ }^{3} \mathrm{H}$ was compiled based on the three closest stations of the IAEA-GNIP -in Flagstaff, Arizona $\left(35^{\circ} 7^{\prime} 48^{\prime \prime} \mathrm{N}\right.$ and $\left.111^{\circ} 40^{\prime} 12^{\prime \prime} \mathrm{W}\right)$, Santa Maria, California $\left(34^{\circ} 53^{\prime} 0^{\prime \prime} \mathrm{N}\right.$ and $\left.12^{\circ} 27^{\prime} 0^{\prime \prime} \mathrm{W}\right)$ and Chihuahua, Mexico $\left(28^{\circ} 37^{\prime} 48^{\prime \prime} \mathrm{N}\right.$ and $\left.106^{\circ} 4^{\prime} 12^{\prime \prime} \mathrm{W}\right)$ by taking the inverse-distance-squared weighted interpolation of the annual average input values of the three stations (Figure 9). The sampling records for Flagstaff, Santa Maria and Chihuahua cover 1961 to 1976, 1961 to 1976 and 1962 to 1988, respectively. For input values after 1988, an annual decrease of the tritium activity of $5.5 \%$ was calculated [14,21]. This is consistent with data from Eastoe [50], who reports an average concentration of 5 TU in rainwater for the Tucson basin from 1992-1998, and data from Harris [51] who reports for the Safford basin, Arizona, ${ }^{3} \mathrm{H}$ levels during the winter of 1994-1995 of 2-3 TU and surface water containing 8-10 TU, which reflect the remaining effects of the atomic bomb tritium spike. The current level in rainwater is estimated to be in the order of 2 to $3 \mathrm{TU}$.

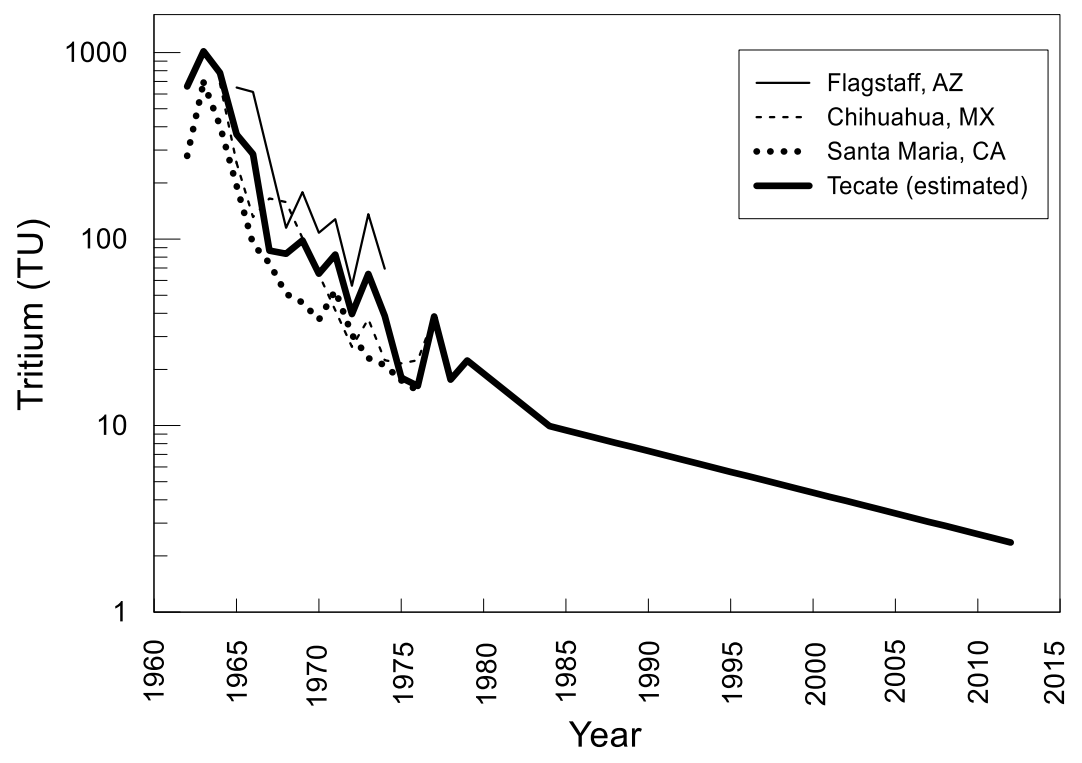

Figure 9. Estimated input function of tritium using the IAEA-GNIP network data of the three closest stations Santa María (California), Flagstaff (Arizona), and Chihuahua (Mexico).

The relation between ${ }^{14} \mathrm{C}$ and ${ }^{3} \mathrm{H}$ shows two different trends (Figure 10a). Most samples in the study site show a narrow range of ${ }^{3} \mathrm{H}$ activity (1.8-2.2 TU), suggesting that recharge from recent years is significant. The exceptions are samples No. 14 and 24 . These two samples have relatively high ${ }^{3} \mathrm{H}$ activities (5.5 and 7.4 TU, respectively), and are related to groundwater mixing with surface water from the Las Auras and Carrizo dams, as mentioned earlier. In general, the high ${ }^{14} \mathrm{C}$ values $(>90 \mathrm{pMC})$ confirm recent recharge and little or no mixture with other older waters. Samples with the highest ${ }^{14} \mathrm{C}$ values, in the order of $108 \mathrm{pMC}$, suggest a recirculation of water in Tecate Valley and consequently a major contribution of bomb-influenced water from the 1950s and 1960s. Carbon-13 ratios vary from -15.4 to $-8.7 \%$. There is a general trend of increasing $\delta^{13} \mathrm{C}$ ratio with increasing ${ }^{14} \mathrm{C}$ values, with the exception of sample No. 14, which is more enriched (Figure 10b). If the carbon-containing rocks were an important source for chemical reactions, the relation would be inverse. Also, the general 
distance between $\delta^{13} \mathrm{C}$ values in groundwater and limestone indicate none or minimal contribution of carbonate rocks. Sample No. 25 and 26 are located in La Rumorosa Valley where marmolized Paleozoic limestone is mined in open quarries. However, the mentioned water samples apparently indicate no influence from carbonate-bearing rocks.
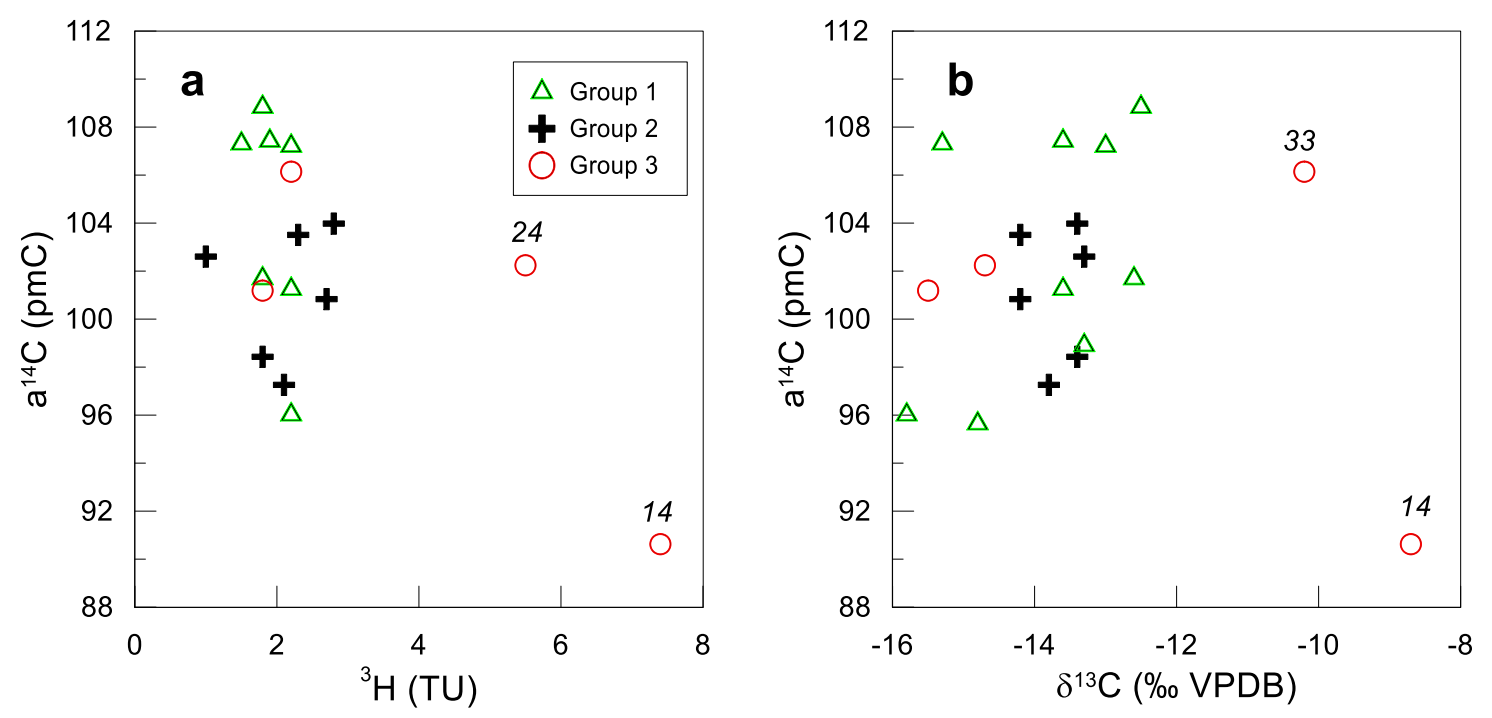

Figure 10. Isotope relationships: (a) ${ }^{14} \mathrm{C}$ vs. ${ }^{3} \mathrm{H}$; (b) ${ }^{14} \mathrm{C}$ vs. $13 \mathrm{C}$.

For radiocarbon age dating two different approaches were used, that gave similar results. According to both, the Pearson model and Fontes and Garnier model, all waters are modern $(<1000$ years old). In general, any estimation is rather uncertain because the age is sensitive to initial values of soil $\delta^{13} \mathrm{C}$ and $\mathrm{a}^{14} \mathrm{C}$. However, testing these models with changed soil $\delta^{13} \mathrm{CCO}_{2}$ values of $\pm 2 \%$ results does not change the results. Similarly, a change of initial ${ }^{14} \mathrm{C}$ activity for $\pm 3 \mathrm{pMC}$ produces the same result. Still, unless there is field data available for soil ${ }^{13} \mathrm{C}$, it is impossible to estimate more accurate groundwater ages. Independently, it can be stated that practically all samples are modern water, and there is no evidence of admixture of fossil groundwater.

With tritium it was possible to perform a semi-quantitative dating approach. This means that groundwater is modern (post-1952), when ${ }^{3} \mathrm{H}$ is larger than the detection limit and ${ }^{14} \mathrm{C}$ is higher than $99 \mathrm{pMC}$; it is pre-modern when ${ }^{3} \mathrm{H}$ is not detected and ${ }^{14} \mathrm{C}$ is below $99 \mathrm{pMC}$; and it is mixed when ${ }^{3} \mathrm{H}$ is detected and ${ }^{14} \mathrm{C}$ is below $99 \mathrm{pMC}$. In accordance with this preliminary classification, thirteen samples $(50 \%)$ were modern, two $(8 \%)$ were pre-modern, and four $(15 \%)$ represent a mix of pre-modern and post-modern, while the rest (seven samples; $27 \%$ ) were not measured.

\section{Discussion}

The joint interpretation of hydrogeological, chemical and isotopic data shows that local groundwater recharge for Tecate Valley is derived from local rainfall and mountain-front infiltration. Groundwater in this unconfined aquifer flows in a western direction at a depth of 5 to $30 \mathrm{~m}$ below ground. It is a mixed- $\mathrm{HCO}_{3}$ to $\mathrm{Na}-\mathrm{HCO}_{3}$ water type with ${ }^{3} \mathrm{H}$ and ${ }^{14} \mathrm{C}$ concentrations of $1.0-2.8 \mathrm{TU}$ and 97.3-104 pMC, respectively. It is characterized by relatively high mineralization $\left(1424 \mu \mathrm{sm}^{-1}\right)$, nitrate (1.9-35.5 $\left.\mathrm{mg} \mathrm{L}^{-1}\right)$ and sulfate $\left(56-275 \mathrm{mg} \mathrm{L}^{-1}\right)$ contents. These data indicate that infiltration from urban area have deteriorated water quality to a degree that it is not potable or close to not drinkable in $76 \%$ of the samples due to the high mineralization. Groundwater from southwest of the Tecate Valley shows admixture of water from the El Carrizo and Las Auras dams. These waters derive from the Río Colorado-Tijuana aqueduct, which are discharged on a riverbed $6 \mathrm{~km}$ away from the El Carrizo dam. Like water from the Colorado River with $\delta^{18} \mathrm{O}=-12.6$ and $\delta^{2} \mathrm{H}=-102.4$ [52] they have significantly more depleted isotope compositions, and high $\mathrm{Cl}^{-}$and $\mathrm{SO}_{4}^{2-}$ concentrations, but very low $\mathrm{NO}_{3}^{-}[52,53]$. 
The ${ }^{3} \mathrm{H}$ values in these samples indicate post-bomb waters. Finally, groundwater from the La Rumorosa Valley at the eastern portion of the study area-a mixed- $\mathrm{HCO}_{3}$ to $\mathrm{Na}-\mathrm{HCO}_{3}$ type-is shallow water, but it has a lower mineralization than Tecate Valley, as well as lower $\mathrm{SO}_{4}^{2-}$ and $\mathrm{Cl}^{-}$concentrations, but similar high $\mathrm{NO}_{3}^{-}$contents. Groundwater from this area is $5-15 \mathrm{~m}$ below ground. Samples from this aquifer are chemically and isotopically similar to those from Tecate Valley. Overall, $64 \%$ of the analyzed samples (mainly the waters of Groups 2 and 3) have nitrate concentrations higher than the threshold value ( $3 \mathrm{mg} / \mathrm{L})$ for anthropogenic influence [40], which suggests nitrate contamination due to infiltration of sewage water, septic deposits, etc. Similarly, $40 \%$ of the water samples showed nitrate levels above the Mexican standard $\left(10 \mathrm{mg} / \mathrm{L}\right.$ as $\left.\mathrm{NO}_{3}\right)$ for drinking water [54], indicating that groundwater should be pretreated for nitrate removal before it can be supplied to population.

The presence of ${ }^{3} \mathrm{H}$ in groundwater evidences that recharge has occurred post-1950. Thus, the groundwater is considered modern. Carbon-14 does match in all samples with this trend. Sample No. 32, for example, reflects initial conditions according to hydrogeological settings. Its $\mathrm{P}_{\mathrm{CO} 2}$ is $10^{-1.9}$ which is similar to soil [14]. This sample has a low alkalinity $\left(\mathrm{HCO}_{3}^{-}=201 \mathrm{mg} \mathrm{L}^{-1}\right)$, and a trend of calcite dissolution $\left(\mathrm{SI}_{\text {calcite }}=-0.6\right.$ ) occurring under open conditions (Table 1, Figure 6). The $\delta^{13} \mathrm{C}$ value of this sample $(-15.5 \%)$ indicates that the $\delta^{13} \mathrm{Csoil} \mathrm{CO}_{2}$ is approximately $-25 \%$ under the given $\mathrm{pH}$ and temperature conditions $\left(7.1 \mathrm{pH}\right.$ units and $\left.18^{\circ} \mathrm{C}\right)$. This is congruent with the initial estimate for $\mathrm{C} 3$ plants (from -20 to $-30 \%$ ) considering a vegetation of coastal sage scrub, chaparral, pine forest, oak forest, and desert scrub.

The travel time of groundwater in wells and springs is indicative of its vulnerability to pollution. The different geochemical ages eventually can be used to evaluate chemical differences in groundwater [20]. In our case, all samples are modern and thus vulnerable, however, no correlation between $\mathrm{NO}_{3}^{-}$or other chemical components concentration with the age of the samples was seen. Thus, it is suggested that the differences in contaminant concentrations are a question of geographic location rather than age differences.

\section{Conclusions}

Human processes associated with urbanization, industry and agriculture have resulted in deterioration in the surface and groundwater quality in the arid Tecate area during the last several decades. Contamination has reduced the usable groundwater supply and made local residents and commercial enterprises more dependent on imported water from the Colorado River. In this study, groundwater flow processes have been investigated using chemical and isotopic evidence. Natural and anthropogenic sources of groundwater contamination have been inspected in addition to hydrogeochemical characterization in relation to groundwater flow.

Groundwater recharge occurs through mountain-block and mountain-front recharge at higher elevations of the ranges around Sierra Juarez and the Laguna Mountains and local rainfall. Groundwater of the unconfined, alluvial aquifer system is of $\mathrm{Na}$ - to mixed- $\mathrm{HCO}_{3}$ type and indicates recent infiltration with little evolution and no mixture with hydrothermal fluids. As groundwater moves rapidly from the hills to the valleys, it acquires salinity, specifically, bicarbonate, sodium, chloride, calcium, sulfate, and magnesium and nitrate, in order of importance. The origin of bicarbonate, sodium, calcium and magnesium ions is primarily incongruent weathering of rock-forming silicate minerals.

There are also human impacts on groundwater chemistry that show spatial differences. Groundwater from the central and northeastern rural area have the lowest mineralization and temperatures, predominant water-rock interactions and nearly pristine conditions. Groundwater from urban Tecate and Rumorosa valleys have higher mineralization, with nitrate originating from urban wastewaters, residual solids and agricultural runoff from fertilizers, livestock manure and/or septic tanks in rural areas. Finally, groundwater from the southwestern portion of the study area are affected by mixtures with surface water from the lower Colorado river, with deterioration in water quality with regard to chloride and sulfate. 
The general trend of a decrease in water quality has until now, scarcely been documented for the last three decades. It is expected that this trend will continue as industrial, and agricultural activites increase and populations grow according to the "business as usual" model. The groundwater system consisting of small unconfined units are highly vulnerable and groundwater sources need to be protected. Additional studies are necessary to define priority areas for groundwater protection. A water quality monitoring program has to be established in order to elaborate a groundwater management program.

Author Contributions: This study was designed by J.M. The hydrogeological framework was developed by J.A.T.-M. Geochemical interpretation was performed by J.M. with help from L.W.D., M.V.E. and A.M. Artwork and formatting was performed by J.A.T.-M. All co-authors contributed to the writing of this manuscript.

Funding: We would like to acknowledge the funding received from Engineering and Science School of Tecnológico de Monterrey via Water Science and Technology research group.

Acknowledgments: Arturo Hernandez-Antonio and Carol Tamez-Melendez are thanked for performing the field work. Technical assistance from Rogelio Ledesma-Ruiz is appreciated. The local water and sanitation service (Comisión Estatal de Servicios Públicos de Tecate) is thanked for facilitating access to public water wells. The three anonymous reviewers are thanked for their comments. Part of this study was presented at the 15th Water-Rock Interaction International Symposium, WRI-15 [47].

Conflicts of Interest: The authors declare no conflict of interest.

\section{References}

1. Castro-Ruíz, J.L. Tecate's water supply. In Tecate, Baja California: Realities and Challenges in a Mexican Border Community; Ganster, P., Cuamea Velázquez, F., Castro Ruiz, J.L., Villegas, A., Eds.; SDSU Press: San Diego, CA, USA, 2002.

2. Instituto Tecnológico y de Estudios Superiores de Monterrey. Estudio hidrogeológico de los acuíferos Las Palmas, Tecate y Rumorosa-Tecate; Instituto Tecnológico y de Estudios Superiores de Monterrey: Monterrey, Mexico, 2017.

3. Wakida, F.T.; Lara-Ruiz, D.; Temores-Peña, J.; Rodriguez-Ventura, J.G.; Diaz, C.; Garcia-Flores, E. Heavy metals in sediments of the Tecate River, Mexico. Environ. Geol. 2008, 54, 637-642. [CrossRef]

4. Lüderitz, V.; Gerlach, F.; Jüpner, R.; Calleros, J.; Pitt, J.; Gersberg, M.; Ju, R. Biological Assessment of Tecate Creek (U.S.-Mexico) with Special Regard to Self-Purification with Special Regard to Self-Purification. Bull.-South. Calif. Acad. Sci. 2005, 104, 1-13.

5. Eckstein, G.E. Rethinking Transboundary Ground Water Resources Management: A Local Approach along the Mexico-US Border. Georg. Int. Environ. Law Rev. 2013, 25, 95-128.

6. Michel, S.M. Rediscovering the Tecate river. In Urban Rivers in Tecate and Tijuana: Strategies for Sustainable Cities; Michel, S.M., Graizbord, C., Eds.; SCERP and IRSC publications: San Diego, CA, USA, 2002.

7. Sanchez, V.; Cortez-Lara, A.A. Minute 319 of the International Boundary and Water Commission between the US and Mexico: Colorado River binational water management implications. Int. J. Water Resour. Dev. 2015, 31, 17-27. [CrossRef]

8. Comisión Nacional del Agua. Determinación de la Disponibilidad de Agua en el Acuífero Tecate (0202), Estado de Baja California; Comisión Nacional del Agua: México City, México, 2015.

9. Comisión Nacional del Agua. Reactivación de Redes de Monitoreo Piezométricas de los Acuíferos de Valle Chico-San Pedro Mártir, Tecate y Laguna Salada en el Estado de Baja California; Comisión Nacional del Agua: México City, México, 2009.

10. Comisión Nacional del Agua. Estudio de actualización de mediciones piezométricas en los Acuíferos: El Rosario, San Rafael, Santo Tomás, Tecate y Laguna Salada, en el estado de Baja California; Comisión Nacional del Agua: México City, México, 2007.

11. Cook, P.G.; Solomon, D.K. Recent advances in dating young groundwater: Chlorofluorocarbons ${ }^{3} \mathrm{H} /{ }^{3} \mathrm{He}$ and ${ }^{85} \mathrm{Kr}$. J. Hydrol. 1997, 191, 245-265. [CrossRef]

12. Newman, B.D.; Osenbrück, K.; Aeschbach-Hertig, W.; Solomon, D.K.; Cook, P.; Rózánski, K.; Kipfer, R. Dating of "young" groundwaters using environmental tracers: Advantages, applications, and research needs. Isot. Environ. Health Stud. 2010, 46, 259-278. [CrossRef] [PubMed]

13. Eastoe, C.J.; Watts, C.J.; Ploughe, M.; Wright, W.E. Future use of tritium in mapping pre-bomb groundwater volumes. Ground Water 2012, 50, 87-93. [CrossRef] [PubMed] 
14. Clark, I.D.; Fritz, P. Environmental Isotopes in Hydrogeology; CRC Press: Boca Raton, FL, USA, 1997; ISBN 1566702496.

15. Li, J.; Liu, J.; Pang, Z.; Wang, X. Characteristics of Chemistry and Stable Isotopes in Groundwater of the Chaobai River Catchment, Beijing. Procedia Earth Planet. Sci. 2013, 7, 487-490. [CrossRef]

16. Kalin, R.M. Radiocarbon Dating of Groundwater Systems. In Environmental Tracers in Subsurface Hydrology; Springer: Boston, MA, USA, 2000; pp. 111-144. ISBN 978-1-4613-7057-4.

17. Vogel, J.C. Vogel Carbon-14 dating of groundwater. Isot. Hydrol. 1970, 1970, 225-239.

18. Fontes, J.-C.; Garnier, J.-M. Determination of the initial 14C activity of the total dissolved carbon: A review of the existing models and a new approach. Water Resour. Res. 1979, 15, 399-413. [CrossRef]

19. Ingerson, E.; Pearson, F.J. Estimation of age and rate of motion of groundwater by the 14C-method. Recent Res. Fields Atmos. Hydrosph. Nucl. Geochem. 1964, 263-283.

20. Mahlknecht, J.; Merchán, D.; Rosner, M.; Meixner, A.; Ledesma-Ruiz, R. Assessing seawater intrusion in an arid coastal aquifer under high anthropogenic influence using major constituents, $\mathrm{Sr}$ and B isotopes in groundwater. Sci. Total Environ. 2017, 587-588, 282-295. [CrossRef] [PubMed]

21. Horst, A.; Mahlknecht, J.; Merkel, B.J.; Aravena, R.; Ramos-Arroyo, Y.R. Evaluation of the recharge processes and impacts of irrigation on groundwater using CFCs and radiogenic isotopes in the Silao-Romita basin, Mexico. Hydrogeol. J. 2008, 16, 1601-1614. [CrossRef]

22. SMN-Servicio Meteorológico Nacional Información Climatológica. Available online: http:/ /smn.conagua. gob.mx/es/climatologia/informacion-climatologica (accessed on 20 April 2018).

23. Thomas, W. Mountain block recharge in Santo Tomas Valley, Baja California, Mexico, Centro de Investigación Científica y de Educación Superior de Ensenada. In Proceedings of the 2009 AGU Fall Meeting Abstracts, San Francisco, CA, USA, 14-18 December 2009.

24. Wilson, J.L.; Guan, H. Mountain-block hydrology and mountain-front recharge. In Groundwater Recharge in a Desert Environment: The Southwestern United States; American Geophysical Union: Washington, DC, USA, 2004; pp. 113-137.

25. Comisión Estatal de Servicios Públicos Tijuana-Sistema Tecate. Informe Hidrogeológico de la Cuenca del Arroyo Tecate; Comisión Estatal de Servicios Públicos Tijuana-Sistema Tecate: Tijuana, Mexico, 1988.

26. Servicio Geológico Mexicano. Carta Geológica-Minera Tijuana I11-11; Servicio Geológico Mexicano: Pachuca, Mexico, 2003.

27. American Public Health Association (APHA). Standard Methods for the Examination of Water and Wastewater, 22nd ed.; APHA/AWWA/WEF: Washington, DC, USA, 2012; ISBN 9780875530130.

28. Östlund, H.G.; Werner, P.E. The Electrolytic Enrichment of Tritium and Deuterium for Natural Tritium Measurements. In Tritium in the Physical and Biological Sciences; International Atomic Energy Agency: Viena, Austria, 1962; Volume 1, pp. 95-105.

29. McCrea, J.M. On the Isotopic Chemistry of Carbonates and a Paleotemperature Scale. J. Chem. Phys. 1950, 18, 849-857. [CrossRef]

30. Ward, J.H. Hierarchical grouping to optimize an objective function. J. Am. Stat. Assoc. 1963, 58, $236-244$. [CrossRef]

31. Parkhurst, D.L.; Appelo, C.A.J. Description of Input and Examples for PHREEQC Version 3-A Computer Program for Speciation, Batch-Reaction, One-Dimensional Transport, and Inverse Geochemical Calculations; U.S. Geological Survey Techniques and Methods: Denver, CO, USA, 2013.

32. Pearson, F.J.; Hanshaw, B.B. Sources of dissolved carbonate species in groundwater and their effects on carbon-14 dating. In Isotope hydrology 1970; International Atomic Energy Agency: Viena, Austria, 1970; pp. 271-285.

33. Plummer, L.N.; Prestemon, E.C.; Parkhurst, D.L. An Interactive Code (NETPATH) for Modeling Net Geochemical Reactions along a Flow Path; USGS Earth Science Information Center: Reston, VA, USA, 1991.

34. Plummer, L.N.; Glynn, P.D. Radiocarbon dating in groundwater systems. In Isotope Methods for Dating Old Groundwater; International Atomic Energy Agency: Viena, Austria, 2013; pp. 33-89. ISBN 978-92-0-137210-9.

35. Ganster, P. Tecate: Challenges for the Twenty-First Century. In Tecate, Baja California: Realities and Challenges in a Mexican Border Community; Ganster, P., Cuamea Velázquez, F., Castro Ruiz, J.L., Villegas, A., Eds.; SDSU Press: San Diego, CA, USA, 2002.

36. Vogel, J.C.; Grootes, P.M.; Mook, W.G. Isotopic fractionation between gaseous and dissolved carbon dioxide. Zeitschrift für Physik 1970, 230, 225-238. [CrossRef] 
37. Mook, W.G.; Bommerson, J.C.; Staverman, W.H. Carbon isotope fractionation between dissolved bicarbonate and gaseous carbon dioxide. Earth Planet. Sci. Lett. 1974, 22, 169-176. [CrossRef]

38. Deines, P.; Langmuir, D.; Harmon, R.S. Stable carbon isotope ratios and the existence of a gas phase in the evolution of carbonate ground waters. Geochim. Cosmochim. Acta 1974, 38, 1147-1164. [CrossRef]

39. Appelo, C.A.J.; Postma, D. Geochemistry, Groundwater and Pollution, 2nd ed.; A.A. Balkema Publishers: Leiden, The Netherlands, 2005.

40. Pastén-Zapata, E.; Ledesma-Ruiz, R.; Harter, T.; Ramírez, A.I.; Mahlknecht, J. Assessment of sources and fate of nitrate in shallow groundwater of an agricultural area by using a multi-tracer approach. Sci. Total Environ. 2014, 470-471, 855-864. [CrossRef] [PubMed]

41. Morán-Ramírez, J.; Ledesma-Ruiz, R.; Mahlknecht, J.; Ramos-Leal, J.A. Rock-water interactions and pollution processes in the volcanic aquifer system of Guadalajara, Mexico, using inverse geochemical modeling. Appl. Geochem. 2016, 68, 79-94. [CrossRef]

42. Kuldip-Singh; Hundal, H.S. Dhanwinder-Singh Geochemistry and assessment of hydrogeochemical processes in groundwater in the southern part of Bathinda district of Punjab, northwest India. Environ. Earth Sci. 2011, 64, 1823-1833. [CrossRef]

43. Patterson, R.A. Domestic Wastewater and the Sodium Factor. In Site Characterization and Design of On-Site Septic Systems; ASTM International: West Conshohocken, PA, USA, 1997; pp. 23-35.

44. Esteller, M.V.; Kondratenko, N.; Expósito, J.L.; Medina, M.; Martin del Campo, M.A. Hydrogeochemical characteristics of a volcanic-sedimentary aquifer with special emphasis on Fe and Mn content: A case study in Mexico. J. Geochem. Explor. 2017, 180, 113-126. [CrossRef]

45. Rozanski, K.; Araguás-Araguás, L.; Gonfiantini, R. Isotopic Patterns in Modern Global Precipitation. In Climate Change in Continental Isotopic Records; Swart, P.K., Lohmann, K.C., Mckenzie, J., Savin, S., Eds.; American Geophysical Union: Washington, DC, USA, 1993; pp. 1-36. ISBN 0-87590-037-2.

46. Kretzschmar, T.G.; Frommen, T. Stable Isotope Composition of Surface and Groundwater in Baja California, Mexico. Procedia Earth Planet. Sci. 2013, 7, 451-454. [CrossRef]

47. Ledesma-Ruiz, R.; Mahlknecht, J. Geochemical and Isotopic Characterization of Groundwater in Tecate, Baja California, Mexico. Procedia Earth Planet. Sci. 2017, 17, 516-519. [CrossRef]

48. Peng, H.; Mayer, B.; Harris, S.; Krouse, H.R. The influence of below-cloud secondary effects on the stable isotope composition of hydrogen and oxygen in precipitation at Calgary, Alberta, Canada. Tellus B Chem. Phys. Meteorol. 2007, 59, 698-704. [CrossRef]

49. Wassenaar, L.I.; Van Wilgenburg, S.L.; Larson, K.; Hobson, K.A. A groundwater isoscape ( $\delta \mathrm{D}, \delta 18 \mathrm{O})$ for Mexico. J. Geochem. Explor. 2009, 102, 123-136. [CrossRef]

50. Eastoe, C.J. Isotopic Data for Tucson Basin: Summary of Work to 1998; Laboratory of Isotope Geochemistry: Tucson, AZ, USA, 1998.

51. Harris, R.C. Tritium as a Tracer of Groundwater Sources and Movement in the Safford Basin, Graham County, Arizona; Arizona Geological Survey: Tucson, AZ, USA, 2000.

52. Daesslé, L.W.; van Geldern, R.; Orozco-Durán, A.; Barth, J.A.C. The 2014 water release into the arid Colorado River delta and associated water losses by evaporation. Sci. Total Environ. 2016, 542, 586-590. [CrossRef] [PubMed]

53. Daesslé, L.W.; Orozco, A.; Struck, U.; Camacho-Ibar, V.F.; van Geldern, R.; Santamaría-del-Angel, E.; Barth, J.A.C. Sources and sinks of nutrients and organic carbon during the 2014 pulse flow of the Colorado River into Mexico. Ecol. Eng. 2017, 106, 799-808. [CrossRef]

54. Secretaria de Salud. NORMA Oficial Mexicana NOM-127-SSA1-1994, Salud Ambiental, Agua Para uso y Consumo Humano-Limites Permisibles de Calidad y Tratamientos a Que Debe Someterse el Agua Para su Potabilización; Secretaria de Salud: Mexico City, Mexico, 1994; pp. 1-7.

(C) 2018 by the authors. Licensee MDPI, Basel, Switzerland. This article is an open access article distributed under the terms and conditions of the Creative Commons Attribution (CC BY) license (http://creativecommons.org/licenses/by/4.0/). 